\title{
Squirrel monkey responses to information from social demonstration and individual exploration using touchscreen and object choice tasks
}

\author{
Elizabeth Renner ${ }^{\text {Corresp., } 1}$, Mark Atkinson ${ }^{1}$, Christine A. Caldwell ${ }^{1}$ \\ 1 Psychology, Faculty of Natural Sciences, University of Stirling, Stirling, United Kingdom \\ Corresponding Author: Elizabeth Renner \\ Email address: elizabeth.renner@stir.ac.uk
}

We aimed to study whether a non-human primate species responded differently to information acquired socially compared with that acquired individually. To do so, we attempted to train squirrel monkeys to perform binary discriminations. These involved exposure to either social information (human or puppet demonstrator performs an initial "information trial") or individual exploration (monkey performs information trial as well as subsequent test trials). In Experiment 1 we presented the task on a touchscreen tablet. Only one monkey appeared to learn the significance of the information trial, and across the group there was no improvement in performance over sessions. The proficient individual showed little evidence of successful transfer to three-way discrimination problems, suggesting limited representation of the task structure. In Experiment 2 we used a logically identical task, presented as a physical object choice (inverted cups concealing a food reward). No monkeys learned to use the information trial cues, and success again did not increase over sessions. We concluded that the monkeys' poor performance in Experiment 1 was not attributable to the mode of presentation (touchscreen), but reflected real difficulties with mastering the task structure. For both experiments, we analysed the monkeys' spontaneous responses to the different trial types (social-win, social-lose, individual-win, and individual-lose). We found that monkeys had a tendency to repeat selections made during the information trial, whether these were made by themselves or by a demonstrator. This tendency to repeat was observed even following lose trials (i.e. when incorrect). Apparent "success" following win trials was probably largely an artefact of behavioural inertia (individual learning conditions) and stimulus enhancement (social learning conditions), rather than sensitivity to the reward cues associated with that stimulus. Although monkeys did respond somewhat differently (more repeats) following win trials, compared with lose trials, this was no more apparent in the object choice task than the touchscreen task, again suggesting that the less ecologically valid presentation medium did not actively disrunt notential for learning the discrimination rule. Both 
touchscreen and physical object choice tasks appear to be valid methods to study learning in squirrel monkeys, with neither method giving a clear performance advantage over the other. However, this population did not master the contingencies in these tasks. 


\section{Squirrel monkey responses to information from social}

\section{3 demonstration and individual exploration using touchscreen}

\section{4 and object choice tasks}

5

6 Elizabeth Renner ${ }^{1}$, Mark Atkinson ${ }^{1}$, Christine A. Caldwell ${ }^{1}$

7

8 Psychology, Faculty of Natural Sciences, University of Stirling, United Kingdom

9

10 Corresponding Author:

11 Elizabeth Renner ${ }^{1}$

12 Psychology, Faculty of Natural Sciences, University of Stirling, Stirling, FK9 4LA, United

13 Kingdom

14 Email address: elizabeth.renner@stir.ac.uk 
16 Abstract

17

We aimed to study whether a non-human primate species responded differently to information acquired socially compared with that acquired individually. To do so, we attempted to train squirrel monkeys to perform binary discriminations. These involved exposure to either social information (human or puppet demonstrator performs an initial "information trial") or individual exploration (monkey performs information trial as well as subsequent test trials). In Experiment 1 we presented the task on a touchscreen tablet. Only one monkey appeared to learn the significance of the information trial, and across the group there was no improvement in performance over sessions. The proficient individual showed little evidence of successful transfer to three-way discrimination problems, suggesting limited representation of the task structure. In Experiment 2 we used a logically identical task, presented as a physical object choice (inverted cups concealing a food reward). No monkeys learned to use the information trial cues, and success again did not increase over sessions. We concluded that the monkeys' poor performance in Experiment 1 was not attributable to the mode of presentation (touchscreen), but reflected real difficulties with mastering the task structure. For both experiments, we analysed the monkeys' spontaneous responses to the different trial types (social-win, social-lose, individual-win, and individual-lose). We found that monkeys had a tendency to repeat selections made during the information trial, whether these were made by themselves or by a demonstrator. This tendency to repeat was observed even following lose trials (i.e. when incorrect). Apparent "success" following win trials was probably largely an artefact of behavioural inertia (individual learning conditions) and stimulus enhancement (social learning conditions), rather than sensitivity to the reward cues associated with that stimulus. Although monkeys did respond 
39 somewhat differently (more repeats) following win trials, compared with lose trials, this was no

40 more apparent in the object choice task than the touchscreen task, again suggesting that the less

41 ecologically valid presentation medium did not actively disrupt potential for learning the

42 discrimination rule. Both touchscreen and physical object choice tasks appear to be valid

43 methods to study learning in squirrel monkeys, with neither method giving a clear performance

44 advantage over the other. However, this population did not master the contingencies in these

45 tasks. 
46

47

48

49

50

51

52

\section{Introduction}

Some explanations of the differences between human culture and the behavioural traditions of other species have suggested that humans are particularly attentive to social information, or even that humans possess specialised cognitive mechanisms for acquiring information from social sources (e.g. Csibra \& Gergely, 2011; Dean et al., 2012; Tennie, Call, \& Tomasello, 2009; Tomasello, Kruger, \& Ratner, 1993). However, it remains difficult to directly compare the effects of exposure to information from a social source with information obtained from one's own experience, since these typically cannot be regarded as equivalent.

Whenever information is potentially generalisable, the inference drawn from observation of another's activity is liable to be very different to that drawn from one's own naïve exploration, even when the action itself is identical. For example, consider being faced with a combination lock on a door, for which you do not know the code. Tapping in a random code yourself (unsuccessfully) will likely lead you to conclude that the code is incorrect. However, observing someone else tapping in a code unsuccessfully might lead you to an entirely different conclusion, or at least to entertain a number of alternative possibilities, many of which would likely entail the information contained within the other's actions having some potential value, despite the unsuccessful outcome. For example, you might conclude that the actor knew the code, but entered one or more digits incorrectly. Alternatively, the code could have been entered correctly but the mechanism was broken, or the actor simply failed to use sufficient force in turning the lock. 
The non-equivalence of information acquired from observation of others, compared with personal experience, arises as a consequence of differential knowledge of the prior behavioural history of the actors in question (complete for ourselves, but usually very limited for others). As a result, in many situations learners would be expected to respond differently to information acquired socially compared with that acquired from individual exploration. Such differential responding would be predicted from either reasoning-based or associative learning accounts, even if the fundamental mechanisms underlying that learning were assumed to be the same.

Theories that propose specialised mechanisms for processing social information in humans would likely predict that humans and non-human primates (NHPs) treat social information somehow differently (Csibra \& Gergely, 2011; Dean et al., 2012; Tennie et al., 2009; Tomasello et al., 1993). However, it is unclear how this difference might manifest in terms of relative responsiveness to information obtained from a social source versus information acquired through one's own personal experience. One possibility is that humans would be more likely (compared with NHPs) to repeat behaviours they had seen others perform, even when the behaviours had not appeared to produce any reward, in a manner that would not occur under individual learning conditions (i.e. following their own performance of an unreinforced response). The tendency of humans to "overimitate" others' irrelevant actions in causal sequences has been well documented (Horner \& Whiten, 2005; Lyons, Young, \& Keil, 2007; Whiten et al., 2016). However, we do not yet know the extent to which humans or NHPs "overimitate" their own unreinforced actions in individual learning conditions; therefore, this first possibility is yet to be empirically assessed. Alternatively, human use of social information might be relatively proficient compared with use of information from direct personal experience, e.g., showing little reduction in learning fidelity, 
92 or possibly even equivalent performance, in relation to the likelihood of repetition of reinforced,

93 over unreinforced, responses. This would be expected to be relative to any such pattern observed

94 in the social and individual learning of NHPs, for whom reduced sensitivity would be predicted

95 for vicarious feedback compared with directly experienced reinforcement contingencies.

97 Thus, it is problematic to evaluate the claim of specialised mechanisms for processing social 98 information in humans based on existing knowledge of the behaviour of either humans or their closest extant evolutionary relatives. This research aims to contribute to the knowledge base upon which such claims could be evaluated. In the studies reported here we attempted to train squirrel monkeys (Saimiri sciureus), a platyrrhine monkey, to learn a discrimination rule using information from either a social demonstration or direct personal experience, with a view to better understanding how these primates respond to information depending on source. In this task the information provided is purely episodic, relating only to that particular problem, and with perfect predictive value. And since the information value of "successful" and "unsuccessful" trials is exactly equivalent across both the social and individual learning conditions, then in principle the performance of any subjects achieving mastery of the task contingencies can be used to evaluate whether source alone affects responses to (otherwise directly comparable) information. We aimed to train monkeys to a pre-set performance criterion which would indicate that they had learned the predictive value of the information available (whether from a social

111 demonstration or their own experience) and could use this to find a rewarded stimulus on 112 subsequent trials involving the same set of stimuli. As well as allowing us to compare the response patterns of those exposed to social information versus personal experience for

114 individuals who reach criterion (i.e. episodic information use), this would also allow us to 
115 compare rates of learning between monkeys assigned to a social learning condition and those

116 assigned to an individual learning condition (i.e. achievement of generalisable task competence).

118 In this task, information trials could consist of selection of either the rewarded or the unrewarded stimulus. Because the task involves binary discriminations, the information received about a response indicates that it should be either repeated (rewarded stimulus selected, or "win" trial) or avoided (unrewarded stimulus selected, or "lose" trial). Successful performance therefore would encompass both reliable re-selection of rewarded stimuli and reliable avoidance of unrewarded stimuli. Within the context of a binary discrimination task, the two information trial types (rewarded and unrewarded) both provide unambiguous information about the location of the reward, and are therefore (in principle) equally informative, assuming the discrimination rule has been learned. We hoped that this format would allow us to examine whether error rates were different following rewarded and unrewarded information trials, and if so, whether this pattern differed between subjects exposed to social information and those learning from their own experience.

Discrimination learning tasks such as the one we used here have been widely employed in studies of animal learning and developmental psychology. These studies have provided evidence of the formation of "learning sets" in children and NHPs (e.g. Harlow, 1949; Levinson \& Reese, 1967). However, most have involved learning either only from personal experience, with information trial and test trial both completed by the participant (e.g. Berman, 1971; Berman, Rane, \& Bahow, 1970, with children), or only from social information, with vicarious observation of the information trial (e.g. Templeton, 1998, with starlings; n.b., we use 
138 "vicarious" throughout to refer to any kind of indirect exposure occurring as a consequence of

139 events that are neither generated, nor directly experienced, by the learner). We know of only one

140 case where similar paradigms have been used to compare performance following exposure to

141 social information with that following individual exploration (Monfardini et al., 2012, with

142 macaques and adult humans). The research questions of Monfardini and colleagues (2012) were

143 rather different from our own, examining how well information was used under significant

144 memory load, with information and test trials separated by several intervening trials.

145 Nonetheless, they found that rewarded and unrewarded trials had differing effects on

146 performance depending on whether they were performed by the subjects themselves or by another monkey. In the individual learning condition, monkeys performed well following a rewarded trial and poorly following an unrewarded trial; but in the social learning condition, the outcome of the information trial did not differentially affect performance.

We first report our attempt to train squirrel monkeys on a touchscreen version of the task

(Experiment 1). Touchscreen tasks have proven useful in making cognition questions feasible to explore in zoo settings (Egelkamp \& Ross, 2019), as touching a screen is a fairly straightforward behaviour to train some animals to do. Such tasks have the additional benefit of allowing researchers to escape the "one-off" nature of object-based tasks (Renner, 2015) and expose an individual to multiple trials, which minimises the effects of outlier days or events and enables repeated measurement of a phenomenon. Squirrel monkeys have been shown to master use of a touchscreen in an experimental setting (Kangas \& Bergman, 2012), though our specific population of monkeys had limited experience of interaction with touchscreens. In Experiment 1, 160 the squirrel monkeys' success rates were very low, and showed little evidence of improvement 
161 over time. To examine whether this was due to the presentation format, we also attempted to

162 train this population using a three-dimensional object-choice version of the task (Experiment 2).

163 The limited success of the squirrel monkeys (on both tasks) meant that we were unable to fully

164 address our original aims of comparing the relative efficacy of social and individual learning and

165 comparing error rates for information trial types. Nevertheless, here we document the monkeys'

166 performance on both tasks. This includes comparisons of the monkeys' performance depending

167 on whether they received information from social demonstrations or their own experience, and of

168 how responses differed following rewarded versus unrewarded information trials. We also

169 compare performance across the two presentation mediums.

170

171

\section{Experiment 1: Touchscreen}

172

173

Materials and Methods

Participants

Approval for this project was provided by the Living Links to Human Evolution Research Centre at RZSS Edinburgh Zoo. At the commencement of this research, a total of 30 squirrel monkeys were housed at the Living Links to Human Evolution Research Centre at Edinburgh Zoo, in two social groups, East and West (MacDonald \& Whiten, 2011). Monkeys had previously been trained with positive reinforcement to enter the research area and allow themselves to be temporarily separated (for a maximum of 15 minutes) from their social group. Research participation was voluntary. The research area for each group consisted of eight cubicles that 
184 allowed to rejoin their social group after indicating a desire to do so by making manual contact

185 with the sliding door. Characteristics of monkeys that participated in this study are presented in

186 Table 1. A total of 13 monkeys ( 8 from the East group and 5 from the West group) passed the

187 touchscreen-training phase of this study (described below). A further 8 monkeys participated in

188 touchscreen training but did not pass the training phase, and 9 monkeys never participated in

189 touchscreen training.

\section{Ethics statement}

192 Ethical approval was granted by the University of Stirling Psychology Ethics Committee.

Animals were not food or water deprived. The research was conducted in line with the guidance provided by the Association for the Study of Animal Behaviour (2015) in Guidelines for the

Treatment of Animals in Behavioural Research and Teaching, and The British Psychological

Society (2012) in Guidelines for Psychologists Working with Animals.

Apparatus

199 A Microsoft Surface 4 touch-sensitive capacitive tablet computer was used to present the training and experimental tasks to the monkeys. The tablet was attached to a rolling projector stand via a metal frame and a mobile arm (Ergotron, Minnesota, USA). The custom program for displaying video of experimental sessions. 
206 Prior to beginning the experiment, monkeys were trained via shaping (Pryor, 2002) to make

207 manual contact with the tablet or the metal frame bordering the touch-sensitive surface of the

208 tablet. Once they reliably did so, they were trained to touch the active area of the screen; next, to

209 touch a large stimulus (approximately $11 \mathrm{~cm}$ in diameter) presented on the screen; and finally, to

210 touch a smaller stimulus (approximately $5.5 \mathrm{~cm}$ in diameter) presented on the screen. When

211 monkeys correctly touched the screen or a stimulus at each respective training level, a visual

212 reward cue appeared, an auditory click sound was played by the tablet, and the monkey was

213 rewarded by the experimenter with a raisin. The visual reward cue for correctly touching training

214 stimuli was a "sunburst" image (see Figure 1B).

215

216 At each of the training levels in which monkeys touched stimuli, they were required to make

217 contact with the relevant part of the screen on 8 or more out of the 10 trials presented in a session

218 and within 30 seconds of the beginning of each trial, and to do this for three consecutive research

219 sessions in order to move on to the next level. To progress to the testing phase, monkeys

220 completed a minimum of eight training sessions with the touchscreen. One monkey progressed to

221 the testing phase after eight training sessions; ten progressed to testing after nine training

222 sessions; two progressed to testing after ten training sessions; and all others engaged in

223 touchscreen training fewer than nine times and therefore never completed training.

\section{Testing procedures}

226 Once monkeys passed the final touchscreen-training level, they were presented with the

228 shapes (circles, triangles, diamonds, pentagons, and hexagons) of various colours (blue, yellow, 
229 black, white, and grey), taking into consideration the limited range of colours platyrrhine

230 monkeys are likely to be able to discriminate (Waitt \& Buchanan-Smith, 2006). Stimulus fill

231 colour, outline colour, and number of sides, as well as background colour, were randomly

232 generated by the task program. For all trials in Stage A, paired stimuli (one on the left, one on the

233 right) were presented; for all trials in Stage B, three stimuli were presented in a horizontal row on

234 the screen. See Figure 1 for an example.

235

236 Stage A. Experimental sessions consisted of a sequence of four problems. Each problem was

237 presented for five trials: a single initial "information trial" (T1) and four subsequent test trials

238 (T2-T5) (see Supplemental Table S1 for details). Although the first test trial (T2), which

239 immediately followed the information trial, was the critical trial from the perspective of the

240 analysis, additional trials were included for every problem as a means to reinforce the monkeys'

241 learning of the overall task structure. Specifically, this was intended to help the monkeys learn

242 that for any given pair of stimuli, only one was rewarded (and the other unrewarded), and that

243 the identity and location of the rewarded stimulus always remained the same within a problem,

244 such that feedback from a selection made on one trial had perfect predictive value regarding

245 reward status for all subsequent trials involving the same pair of stimuli. Stimuli did not change

246 position between trials within a problem.

Initial selections were randomly assigned to be either rewarded or unrewarded. That is, we programmed the task to generate a list such as $[\mathrm{U} R \mathrm{U} \mathrm{R}]$, meaning that the stimulus selected on the information trial of the first problem was unrewarded (regardless of which position on the

251 screen the stimulus occupied), and that the alternative stimulus would then be the rewarded 
252 stimulus. The stimulus selected on the information trial of the second problem was then the

253 rewarded stimulus (regardless of its position), and so on. We programmed the task to ensure that

254 half of information trials were rewarded and half were unrewarded. This was to help prevent

255 monkeys from developing side biases. For example, if a monkey always selected the stimulus on

256 the left in information trials, the left-side stimulus would be the rewarded stimulus only half of

257 the time.

258

259

When a rewarded stimulus was selected, both stimuli disappeared, a sunburst visual reward cue appeared where the rewarded stimulus had been, and an auditory click was generated by the computer. Additionally, when a monkey selected a rewarded stimulus on any trial, they received a raisin. When an unrewarded stimulus was selected, all stimuli disappeared for a time-out of 3 seconds, no auditory cue was emitted, and no raisin was dispensed. The same stimulus, in the same location on the screen, was the rewarded stimulus for an entire problem (i.e. one information trial followed by four test trials using the same pair of stimuli).

Half of the monkeys were assigned to the individual condition, and the other half were assigned to the social condition. In the individual condition, at the beginning of a session, the tablet was placed within a monkey's reaching distance from the cubicle by use of a mobile trolley. Then monkeys themselves selected one of the stimuli in the information trial, and were rewarded (or not) accordingly. They were then allowed to select one of the stimuli on each of the four subsequent test trials. Between problems, the tablet was moved outside of reaching distance for several seconds before again being placed within reaching distance for the next problem. 
275 In the social condition, at the beginning of a session, the tablet was placed outside of a monkey's

276 reaching distance from the cubicle. Monkeys saw a social demonstrator select one of the stimuli

277 in the information trial. The tablet was then placed in reaching distance to allow the monkey to

278 perform the next four test trials. After this, the tablet was moved outside of reaching distance to

279 prepare for the start of the next problem.

280

281 In half of the research sessions, the social demonstrator was the human experimenter, and in the

282 other half, this was a puppet (a plush squirrel monkey toy with some of the stuffing removed;

283 Wild Republic, Twinsburg, Ohio) operated by the experimenter (Figure 2). Researchers have

284 successfully used a puppet demonstrator in a study with children (Wood, Kendal, \& Flynn,

285 2013), a stuffed conspecific demonstrator in a study with birds (Truskanov \& Lotem, 2017), and

286 videos of a human dressed in an ape-like costume in a study with great apes (Krupenye et al.,

287 2016). Because of the previously successful implementation of these methods, we explored

288 whether a puppet demonstrator would induce better performance than a traditional human

289 demonstrator on this task. Previous research also found that a "monkey-like" human

290 demonstrator, who did not solicit monkeys' attention and consumed rewards after rewarded

291 trials, resulted in better subsequent performance than a traditional human demonstrator who

292 solicited monkeys' attention and did not consume rewards (Monfardini, Hadj-Bouziane, \&

293 Meunier, 2014). However, due to the potential for distraction, neither the experimenter nor the

294 puppet received a food reward after selecting the rewarded stimulus on T1.

296 We were initially interested in whether monkeys would successfully transfer from Stage A (two

297 stimuli) to Stage B (three stimuli). Therefore, we established a performance criterion for 
298 advancing from Stage A to Stage B. Criterion was set as correct performance on the second trials

299 (T2s) on three of the four problems (75\%) in a session, in addition to correct performance on T3

300 through $\mathrm{T} 5$ of $75 \%$ or more in a session, for three sessions in a row.

301

302 Stage B. Monkeys that passed criterion (as detailed above) on Stage A commenced Stage B.

303 Testing procedures for Stage B were identical to Stage A with the exception that each problem

304 involved three stimuli, and therefore two of these were unrewarded (and only one rewarded, as

305 per Stage A). Otherwise, testing was structured as in Stage A (i.e. four problems per session, five

306 trials per problem, and consistent reward location for all trials of a given problem).

307

308 During the data collection period (May to November 2016), up to two research sessions were 309 conducted per day. All 13 participating monkeys completed at least 10 testing sessions of Stage

310 A (see Table 1), with the minimum number of sessions being 14 and the maximum being 69.

312 Statistical analysis

313 Statistical analysis was done via logit fit GLMMs using the lme4 package (Bates et al., 2015) in

314 R (R Core Team, 2017). The following fixed effects were included in at least some of the

315 models: session number (scaled and centred), which was used to determine whether monkeys'

316 performance improved with experience; trial number; the source of information (human, puppet,

317 or individual); and the type of information (whether the information trial was a win or a lose;

318 sum coded). Random effects included in at least some of the models were monkey identity

319 (random intercept effect) and session number and information type by monkey (random slope

320 effects); we used models with "maximal” random effects structures (Barr et al., 2013) in the first 
321 instance, removing random slopes followed by random intercepts as necessary to address issues

322 of singular fit or non-convergence. Post hoc analyses were done using the emmeans package

323 (Lenth et al., 2019).

324

325 We used three main response variables: (1) trial success indicates whether the correct stimulus

326

327

328

329

330

331

332

333

334

335

336

337

338

339

340

341

342

343 was selected on a given trial (binary: $1=$ yes; $0=$ no); (2) WSLS (win-stay, lose-shift) indicates which strategy was used on a particular trial in relation to the information trial (binary: $1=$ winstay or lose-shift; $0=$ win-shift or lose-stay); and (3) repeats indicates whether the same stimulus was selected on a given trial as was selected in the information trial (binary: $1=$ repeated; $0=$ not repeated). For Stage A (two stimuli), success and WSLS have the same values. However, for Stage B, these variables may hold different values (i.e. it is possible that, after a lose information trial, a subject can shift and select a second unrewarded stimulus, in which case their success score would be 0 and their WSLS score would be 1).

\section{Results}

37 Although we had hoped to compare the effectiveness of learning from social demonstrations with that of learning from personal experience, in fact only one monkey reached our performance criterion. Aside from the single individual who reached criterion, there was little evidence that the monkeys were learning anything from their increasing experience with the task. Taking correct stimulus choice (success) on T2 as the measure of interest (i.e. re-selection of stimuli that were rewarded in the information trial, and avoidance of stimuli that were unrewarded), overall performance on T2 of the two-stimulus task (Stage A) was slightly greater 
344 than chance, with 56\% T2 success (Table 2; observed success: 1283/2293 trials; expected:

$3451146.5 / 2293, p<0.001$ in a binomial test). Results of the various models are summarised in 346 Table 3.

347

348

Effect of experience (session number)

349

350

351

A GLMM with a response variable of T2 success, a fixed effect of session number (scaled and centred), and a random effect of monkey identity showed that performance accuracy did not improve with increasing session number $(b=0.017, \mathrm{SE}=0.024, \mathrm{Z}=0.72, p=0.47)$. That is, monkeys overall did not improve their task performance with more experience.

\section{Information type, information source, and interactions}

Although the monkeys had not learned the task, it was nonetheless of interest to know how they performed across the different trial types (i.e. individual-win, individual-lose, social-win, and social-lose, with social demonstrations also broken down into human and puppet demonstrations).

To explore this, we built a GLMM with T2 success as the response variable; information type, information source, and an interaction of these two variables as fixed effects; a random intercept effect of monkey identity; and session number as a by-monkey random slope effect. We used Helmert contrasts (in the stats package in R [R Core Team, 2017]) to examine whether performance differed between the three information sources. In Helmert contrasts, the first two levels of a variable are compared to each other, the third level is compared to the mean of the first two levels, and so on (Fox, 2002; Schad et al., 2018). The key comparisons here are (1) 
367 between the two social or vicarious sources of information (human and puppet demonstrations)

368 and (2) between the social and individual sources of information. Therefore, we set the first level

369 as human demonstration, for comparison to the second level, puppet demonstration; and finally,

370 the combined human and puppet levels were compared to the third level, the individual

371 condition. There was a main effect of information type; success after seeing a win trial (68\%

372 success) was significantly higher than that after seeing a lose trial ( $44 \%$ success; $b=0.44, \mathrm{SE}=$

$3730.046, Z=9.6, p<0.001)$. In addition, success after seeing a puppet demonstration $(54 \%)$ was

374 significantly lower than that after seeing a human demonstration $(60 \% ; b=-0.13, \mathrm{SE}=0.063, \mathrm{Z}$

$375=-2.0, p=0.043)$. There was not a significant difference between the combined social

376 conditions (puppet and human) and the individual condition ( $55 \% \mathrm{~T} 2$ success; $b=-0.020, \mathrm{SE}=$

$3770.040, \mathrm{Z}=-0.52, p=0.61)$. However, there was an interaction between information type and

378 source, indicating a difference between the third level of the source variable (individual

379 condition $)$ and the other two levels $(b=0.090, \mathrm{SE}=0.029, \mathrm{Z}=3.1, p=0.002)$. To clarify this

380 interaction, we performed a post hoc analysis using emmeans. This indicated that the

381 disadvantage in performance after lose demonstration trials was greater in the individual

382 condition (after a lose, $40 \%$ success; after a win, $70 \%$ success) than in the two social conditions

383 (after a lose, 49\% success; after a win, 66\% success; Supplemental Table S2).

384

385

Repeating previous selections

386

While T2 success was the main variable of interest, we noted that the pattern of responses

387

possibly indicated a "stay" bias (that is, a tendency to repeat the selection from the previous trial, regardless of whether this followed a win or lose information trial). This raised the possibility that the monkeys were influenced only minimally, if at all, by the presence or otherwise of the 
390 reward cue during the information trial. That is, they may not have been discriminating between

391 win and lose trials at all. In the individual condition the monkeys may have simply had a

392 tendency to repeat their own previous selections, regardless of any feedback obtained. And in the

393 social condition they could have been subject to fairly nonspecific stimulus enhancement effects

394 which drew their attention to previously selected stimuli, again irrespective of whether this had

395 been associated with the reward cue. This would suggest that the monkeys were not using the

396 information trial as "information" at all, if the outcome of the trial did not influence their choice

397 of stimulus.

398

399 Therefore, we analysed another response variable, T2 repeats. This binary variable captured

400 whether, on a given T2, a subject repeated the response (either their own or observed) from the

401 information trial, which was coded as 1 , or switched responses, which was coded as 0 . We used a

402 GLMM with T2 repeats as the response variable; information type, information source, and an

403 interaction of these two variables as fixed effects; a random intercept effect of monkey identity;

404 and session number as a by-monkey random slope effect. We again used Helmert contrasts to

405 examine the effect of information source. Human demonstration was the first level, puppet

406 demonstration was the second level, and the individual condition was the third level. There was a

407 main effect of information type; tendency to repeat after a win trial (68\% repeats) was higher

408 than tendency to repeat after a lose trial $(56 \%$ repeats; $b=0.28, \mathrm{SE}=0.047, \mathrm{Z}=6.0, p<0.001)$.

409 (But note that the proportion of repeats following both information trial types is above $50 \%$

410 [Figure 3].) This indicates a degree of sensitivity to the information in T1, even if the difference

411 is modest. There was no main effect of source, indicating that there were not significant

412 differences in the tendency to repeat between puppet and human demonstrations $(b=-0.065$, SE 
$413=0.063, \mathrm{Z}=-1.0, p=0.31)$ or between vicarious and individual selection of a stimulus $(b=$

$4140.11, \mathrm{SE}=0.065, \mathrm{Z}=1.6, p=0.10)$. The interaction between the information type and the

415 individual vs social conditions was not significant $(b=-0.028, \mathrm{SE}=0.029, \mathrm{Z}=-0.95, p=0.34)$.

416 The interaction between information type and social demonstration type (puppet and human) was

417 non-significant but near the alpha criterion threshold $(b=-0.12, \mathrm{SE}=0.063, \mathrm{Z}=-1.9, p=$

418 0.056). Further analysis of this trend indicates that the tendency to repeat the puppet's selection

419 (after a win, 62\% repeats; after a lose, 53\% repeats) was less influenced by information type than

420 the tendency to repeat the human's selection (after a win, 70\% repeats; after a lose, 50\% repeats).

421

422

Improvement across trials

423 Because the monkeys appeared not to learn the discrimination rule, and showed a significant 424 tendency to repeat selections from the information trial following both win and lose trials, this 425 raised the question of whether the monkeys were sensitive to task feedback at all. We therefore 426 wished to test whether the additional feedback obtained from the monkeys' selections across all

427 trials for any given problem improved their chance of success (since all information trials were

428 followed by not only the critical test trial T2, but also three further trials using the same

429 problem- $-\mathrm{T} 3, \mathrm{~T} 4$, and $\mathrm{T} 5$ - intended to reinforce recognition of the predictive relationship

430 between successive trials using the same pair of stimuli). To determine whether monkeys

431 improved their performance across trials within a problem (as they presumably received

432 confirmatory information about where the rewarded stimulus was or was not), we used a GLMM

433 with success in each trial from $\mathrm{T} 2$ to $\mathrm{T} 5$ as the response variable; trial number as the fixed effect;

434 a random intercept effect of monkey identity; and session number, information type, and their

435 interaction as by-monkey random slope effects. A non-significant trend towards improvement 
436 across trials was demonstrated (Figure $4 ; b=0.036, \mathrm{SE}=0.019, \mathrm{Z}=1.9, p=0.064$ ). It is

437 therefore not possible to conclude that monkeys located the reward more accurately in later than 438 in earlier trials.

439

440

Three-stimulus task (Stage B)

441 A single monkey (Amarilla) met criterion on the two-stimulus task and was tested on the three-

442 stimulus task (Stage B) for 19 sessions. Amarilla remained in the individual condition throughout

443 the study. Despite having reached criterion on the two-stimulus task, Amarilla failed to reach the 444 same performance criterion (of appropriate stay/shift responses on $\mathrm{T} 2$ for $75 \%$ of problems in a 445 session, and appropriate stay/shift responses on T3-T5 for 75\% or more trials in a session, for 446 three sessions in a row) on the three-stimulus task.

447

448 Our original goal in training the monkeys had been to train them to general competency using 449 this discrimination paradigm, so that we could test the use of social information for more taxing 450 stimulus selection problems. However, this was not possible during the data collection period. 451 Therefore Amarilla's testing sessions were terminated when the planned data collection period 452 ended. Despite this, we did have sufficient data from this subject to perform some analyses on 453 how she transferred her Stage A learning to the novel Stage B context. Her T2 win-stay 454 performance in Stage A was 66\% (compared to a chance level of 50\%) and in Stage B was 44\% 455 (compared to a chance level of 33\%). Her T2 lose-shift performance in Stage A was 53\% 456 (compared to a chance level of 50\%) and in Stage B was 71\% (compared to a chance level of $45767 \%$ ). The observed values were compared to chance by use of binomial tests: the only category 
458 of trial in which Amarilla's performance differed from chance was Stage A win-stay (observed:

45948 win-stays/73 win trials in Stage A; expected: $36.5 / 73 ; p=0.0095$ ).

460

461 This pattern of performance led to Amarilla finding the rewarded stimulus (and therefore experiencing the reward cue and receiving food reinforcement) in 59\% of Stage A T2 trials and $38 \%$ of Stage B T2 trials. A summary of the results (showing performance as measured by T2 repeats in order to demonstrate comparisons with chance) is presented graphically in Figure 5.

To determine whether there was a learning effect (i.e. whether her performance improved with

more experience), we used a generalised linear model with T2 WSLS as the dependent variable and session number (scaled and centred) as the fixed effect. This model showed no effect of session number $(b=0.12, \mathrm{SE}=0.45, \mathrm{Z}=0.27, p=0.79)$, indicating that Amarilla's performance did not improve with experience in Stage B.

\section{Conclusion}

In general, the squirrel monkeys did not appear to have learned to use the information trial as a cue that ensured success on the test trial(s). Although one subject reached the criterion and transferred from Stage A to Stage B, the success with which she ultimately did so was limited.

One potential explanation for the monkeys' limited proficiency with the task was the presentation medium. There is evidence that both young children (Barr \& Hayne, 1999) and capuchin monkeys (Anderson, Kuroshima, \& Fujita, 2017) learn better when using threedimensional objects than when viewing videos of a demonstration, a phenomenon termed the 
481 video deficit effect. If squirrel monkeys' performance on the touchscreen task was hampered by

482 something like the video deficit effect, we reasoned that performance might be improved by

483 arrays consisting of three-dimensional objects. Therefore, we designed Experiment 2 to

484 reproduce the contingencies of Experiment 1 but to utilise three-dimensional objects as stimuli.

485 Although we intended to reproduce our procedures from Experiment 1 as closely as possible, we

486 decided to use human demonstrations only in the social condition, dispensing with the puppet.

487 This was due to the results of Experiment 1 suggesting that the monkeys performed, if anything,

488 less well following puppet demonstrations, compared with human ones.

489

490

Experiment 2: Three-dimensional objects

491

492

Experiment 2 was pre-registered at the Open Science Framework (osf.io/phzf2).

493

494

\section{Materials and Methods}

495

496

Participants

497

Approval for this project was provided by the Living Links to Human Evolution Research Centre at RZSS Edinburgh Zoo. The same groups of squirrel monkeys participated in Experiment 2 (see

Table 1 for biographical details); by the beginning of this study, the number of monkeys had grown via births to 35 individuals. A total of 11 monkeys ( 7 from the East group and 4 from the

West group) participated in 10 or more experimental sessions (the number specified in the preregistration as the minimum for their data to be included); a further 8 monkeys participated in the experiment but not enough times for their data to be included ( $<10$ sessions each); and 16 
504 monkeys never participated in the study. The data from one subject (Toomi) were excluded, as

505 outlined in the pre-registration, because she developed a side bias ( $>80 \%$ of selections on the 506 same side).

507

\section{Ethics statement}

509 Ethical approval was granted by the University of Stirling Animal Welfare and Ethical Review 510 Body under proposal number AWERB (16 17) 117. Animals were not food or water deprived.

511 The research was conducted in line with the guidance provided by the Association for the Study 512 of Animal Behaviour (2015) in Guidelines for the Treatment of Animals in Behavioural

513 Research and Teaching, and the British Psychological Society (2012) in Guidelines for

514 Psychologists Working with Animals.

516 Apparatus

517 Objects were small plastic cups $(7 \mathrm{~cm}$ tall $\times 5.5 \mathrm{~cm}$ wide $\times 5.5 \mathrm{~cm}$ deep; Sistema, New Zealand $)$ 518 covered with differently coloured and patterned felt sleeves $(20 \mathrm{~cm}$ long $\times 7 \mathrm{~cm}$ tall $)$ that were 519 attached using strips of Velcro (Figure 6). Objects were presented on top of a mobile trolley. The 520 baiting of the cups was hidden from the monkeys' view with a cardboard occluder. A digital 521 video camera was used to record video of experimental sessions.

\section{Training procedures}

524 In the course of their previous experience in the research facility, prior to taking part in the 525 experiments reported here, the monkeys had been familiarised with a training activity. In this

526 activity, a single raisin was visibly placed under one of two cups, in trials involving a range of 
527 levels of difficulty, and monkeys were then allowed to choose between the cups. Thus, monkeys

528 were already trained to select one of two objects, one of which contained a treat, using their

529 hands. Therefore, no further task training was undertaken before the commencement of

530 Experiment 2. A brief familiarisation session (to allow the monkeys to become familiar with the

531 materials presented to them) involved giving monkeys rewards while the experimenter

532 manipulated the trolley and occluder.

533

534 Testing procedures

535 Testing procedures were similar to those described above for Experiment 1. Each testing session 536 consisted of four problems, and each problem had five trials. For a single problem, two cups,

537 with different sleeves, were placed, open end down, on the rolling trolley. The order of the use of 538 sleeves was random, so that no particular pattern or colour was systematically rewarded or was 539 systematically paired with any other. To begin a problem, the cardboard occluder was placed in 540 front of the cups; a sunflower seed or raisin reward was held above the occluder; and the reward 541 was then lowered behind the occluder and placed under a single cup. During the hiding process, 542 both cups were lifted so that auditory cues would not reveal the location of the reward. Then, the 543 occluder was removed and the information trial was carried out, followed by four test trials.

544 Stimuli remained in the same position for an entire problem. The occluder was brought down in 545 front of the cups between trials, so that re-baiting, if necessary, would not be visible to the 546 monkey.

548 Monkeys remained assigned to the same information source condition they experienced in

549 Experiment 1 (so that if they were in the individual condition in Experiment 1, they remained in 
550 the individual condition in Experiment 2). In the individual condition, monkeys selected one of

551 the cups in the information trial, and were rewarded (or not) accordingly. The setup was then re-

552 baited, and monkeys were then allowed to select a cup on the subsequent test trials by reaching

553 through the window toward it or touching or lifting it. In the social condition, monkeys saw a

554 social demonstrator select one of the stimuli in the information trial: this was always the human

555 experimenter, who did not consume the food reward if the rewarded cup was selected but did

556 remove the reward as if it had been consumed. The setup was then re-baited, and monkeys were

557 allowed to select a cup on the subsequent test trials. As in Experiment 1, the purpose of the

558 additional test trials using the same pair of stimuli was to provide scaffolding to allow the

559 monkeys to learn that the reward remained in the same single location for any given pair of

560 stimuli, although T2 again was the critical trial for the purpose of analysis. Monkeys received a

561 food reward on each trial where they selected the rewarded stimulus.

562

563 Rewards were randomly allocated to the left or right side for a problem, and patterns of lefts and

564 rights were pseudorandomised so that the reward location would not be predictable and so that

565 rewards would occur equally on each side (to help prevent the development of side biases).

566

567 Statistical analysis

568 Statistical analysis was done via logit fit GLMMs using the lme4 package (Bates et al., 2015) in

$569 \mathrm{R}$ (R Core Team, 2017). As in Experiment 1, the main response variables were success, WSLS, 570 and repeats. Fixed effects used in at least some of the models included the session number

571 (centred), trial number, source of information (social or individual; sum coded), and type of

572 information (whether T1 was a win or a lose selection; sum coded). Random effects included 
573 monkey identity and side (left or right) of the rewarded stimulus (random intercept effects);

574 similarly to Experiment 1, we removed random effects as necessary to address issues of singular

575 fit or non-convergence.

576

577 Results

As in Experiment 1, overall success on T2 was slightly greater than chance, with $55 \% \mathrm{~T} 2$ success

(Table 2; observed success: 305 wins/557 trials; expected: $278.5 / 557 ; p=0.027$ in a binomial

581 test).

582

Effect of experience (session number)

584 To evaluate the effect of experience, we used a GLMM with T2 success as the response variable, session number (centred) as the only fixed effect, and monkey identity and side of the rewarded item as random intercept effects ${ }^{1}$. This model showed no significant change in T2 success in relation to session number $(b=0.025, \mathrm{SE}=0.016, \mathrm{Z}=1.6, p=0.12)$; that is, when stimuli were 3D objects, as when they were presented on a touchscreen, monkeys did not improve their task performance with greater experience.

Information type and source

592 The full pre-registered GLMM was built, with T2 WSLS as the response variable; information

593 source (social or individual), information type (win or lose), and their interaction as fixed effects;

\footnotetext{
${ }^{1}$ Due to an oversight, we failed to specify the random effects structure we intended for this model in our the preregistration. This analysis uses the same random effects structure as pre-registered for our main model (see "Information type and source" below). Alternative random effects structures make no difference to the pattern of results we present here.
} 
594 and monkey identity and side of the rewarded item as random intercept effects. This model

595 revealed a main effect of information type (win trial [64\% WSLS] $>$ lose trial [46\% WSLS]; $b=$ $5960.54, \mathrm{SE}=0.097, \mathrm{Z}=5.5, p<0.001)$ and no main effect of source $(b=0.051, \mathrm{SE}=0.12, \mathrm{Z}=$ $5970.44, p=0.66)$. There was an interaction between information type and source $(b=0.55, \mathrm{SE}=$ $5980.098, \mathrm{Z}=5.7, p<0.001)$. To clarify this interaction, we used the emmeans package to perform a 599 post hoc analysis. This indicated that in the social condition, there was no difference in 600 performance after seeing win (54\% WSLS) and lose (55\% WSLS) demonstrations; however, in 601 the individual condition, performance was better after experiencing a win (79\% WSLS) than a 602 lose (31\% WSLS) in the information trial (see Table S3 in the Supplemental Material).

Repeating previous selections

605 We analysed the repeats variable for Experiment 2, following the same logic as for Experiment 1 606 (i.e., to determine whether the monkeys were sensitive to whether they had observed a win or 607 lose trial). Note that analyses using the repeats variable were not pre-registered. We constructed 608 a GLMM with T2 repeats as the response variable; information source, information type, and 609 their interaction as fixed effects; and monkey identity as a random intercept effect. There were 610 main effects of information source and information type, and no interaction between these 611 variables $(b=0.045, \mathrm{SE}=0.097, \mathrm{Z}=0.46, p=0.64)$. The tendency to repeat was stronger in the 612 individual ( $74 \%$ repeats) than the social ( $49 \%$ repeats) condition $(b=0.52, \mathrm{SE}=0.14, \mathrm{Z}=3.8, p$ $613<0.001)$, and the tendency to repeat was stronger after win information trials $(64 \%$ repeats $)$ than 614 lose information trials ( $54 \%$ repeats; $b=0.23, \mathrm{SE}=0.10, \mathrm{Z}=2.3, p=0.020)$ (although note that 615 the tendency to repeat was greater than 50\% even following lose trials). Monkeys' performance 616 using the repeats measure is presented in Figure 7. 
619 Following the same logic of Experiment 1, we examined whether monkeys improved their

620 performance across trials within a problem, as another means of determining whether monkeys

621 were sensitive to the win/lose feedback they received. We built a GLMM with success in each

622 trial from T2 to T5 as the response variable, trial number as the fixed effect, and side of the

623 rewarded item as a random intercept effect. ${ }^{2}$ Trial number did have a significant effect (Figure

624 8), indicating that monkeys more frequently selected the rewarded stimulus as trial number

625 increased $(b=0.092, \mathrm{SE}=0.039, \mathrm{Z}=2.3, p=0.020)$. The magnitude of the effect was modest,

626 with overall success on T2 of 55\% and overall success on T5 of $61 \%$.

\section{Comparison of $2 D$ and $3 D$ presentations}

629 To compare the effect of the presentation mediums used in Experiments 1 and 2, we combined 630 the data sets (using only Stage A data from Experiment 1) and built a GLM with T2 WSLS as

631 the response variable; and information type, information source, presentation medium

632 (touchscreen or cups), and interactions between these variables as fixed effects. ${ }^{3}$ Similarly to the

633 models of the data from the two experiments run separately, this GLM revealed a main effect of

634 information type (win trial $[67 \% \mathrm{WSLS}]>$ lose trial $[45 \% \mathrm{WSLS}] ; b=0.50, \mathrm{SE}=0.053, \mathrm{Z}=$

$6359.6, p<0.001$ ), while the main effects of the variables information source and presentation

636 medium were non-significant (all $p \mathrm{~s}>0.67$ ). There was a significant interaction between

${ }^{2}$ Due to an oversight, we failed to specify the random effects structure we intended for this model in the preregistration. In this analysis, we attempted to use the random effects pre-registered for our main model; however, including monkey identity as a random intercept effect caused the model to have a singular fit. Therefore, only side of the rewarded item was included..

${ }^{3}$ Due to an oversight, we failed to specify the random effects structure we intended for this model in our preregistration. Although we built a model with a random effect of monkey identity, this model had a singular fit, and side of the rewarded item was not an existing variable in the touchscreen data set. This was the reason for use of a GLM. 
637 information type and source $(b=0.33, \mathrm{SE}=0.053, \mathrm{Z}=6.3, p<0.001)$, and a three-way

638 interaction between information type, source, and presentation medium $(b=-0.20, \mathrm{SE}=0.053$,

$639 \mathrm{Z}=-3.8, p<0.001)$. For post hoc analysis of this interaction, see Table S4 in the Supplemental

640 Material. All other interactions were non-significant.

641

642

Repeating previous selections: a comparison of $2 D$ and $3 D$ presentations

643 Additionally, we constructed a non-pre-registered GLMM with T2 repeats as the response

644 variable. This model had fixed effects of information type, information source, presentation

645 medium, and interactions between these variables; and monkey identity as a random intercept

646 effect. This model revealed main effects of information type (more repeats after a win [67\%

647 repeats] than a lose $[55 \%$ repeats $]$ information trial; $b=0.24, \mathrm{SE}=0.053, \mathrm{Z}=4.5, p<0.001)$ and

648 information source (more repeats in the individual condition [66\% repeats] than the social

649 condition $[56 \%$ repeats]; $b=0.34, \mathrm{SE}=0.086, \mathrm{Z}=3.9, p<0.001)$. There was no main effect of

650 presentation medium $(b=-0.017, \mathrm{SE}=0.057, \mathrm{Z}=-0.29, p=0.77)$. However, there was a

651 significant interaction between information source and presentation medium $(b=-0.22, \mathrm{SE}=$

$6520.057, \mathrm{Z}=-3.8, p<0.001$; see Supplemental Material Table S5). All other interactions in this

653 analysis were non-significant. Results are presented graphically in Figure 9.

654

655 Discussion

656

657 In exposing squirrel monkeys to binary discrimination learning problems, we had originally

658 hoped that they would achieve a performance criterion that could be regarded as indicative of

659 having learned the task contingencies, if given experience of multiple sessions each involving 
660 several problems. Establishing task competence would have allowed us to answer certain

661 questions about the monkeys' responses to information acquired from social observation

662 compared with that acquired from their own personal experience. Although we had limited

663 success fulfilling this original aim, we were nonetheless able to analyse the squirrel monkeys'

664 spontaneous reactions to the task (presumably relatively uncontaminated by the effects of

665 reinforcement learning within the task itself). In addition, we were able to compare the relative

666 effectiveness of two alternative mediums of task presentation-2D touchscreen interaction and

667 3D object choice - as well as determine whether the monkeys displayed similar patterns of

668 spontaneous responses across these two task types.

669

670 Overall, the performance of the squirrel monkeys in both tasks was only very slightly above

671 chance. This contrasts with the performance of preschool children in a very similar task

672 (Atkinson et al., 2019), who spontaneously adopted a win-stay, lose-shift strategy and thus

673 demonstrated highly proficient performance with little or no previous task experience. The

674 squirrel monkeys' performance suggests that even a very simple choice task, involving

675 straightforwardly predictive task cues, may be difficult for them to master unless they have

676 extensive training with the paradigm and/or elevated motivation to succeed (such as calorie

677 restriction, which has been used in other studies carried out by different research groups (Kangas

$678 \&$ Bergman, 2012)).

679

680 In addition, the squirrel monkeys did not show performance improvement across sessions; that

681 is, more experience with the tasks did not lead to better performance. Therefore, inasmuch as

682 they performed marginally above chance, this was not due to learning the task-specific 
683 significance of rewarded versus unrewarded information trials, but instead reflected the 684 spontaneous after-effects of having performed an action, or having been exposed to the actions 685 of another, somewhat moderated by whether or not these actions had been associated with 686 rewards and/or reward cues. Although a single monkey did reach our performance criterion on 687 the two-stimulus version of the touchscreen task, her performance upon transfer to the three688 stimulus version of the task indicated that, even in this case, there were limits to her 689 understanding of the task contingencies. The monkey's performance suggested that the fidelity 690 of her responses shifted in line with the change in expected response frequencies based on 691 chance dictated by the increase in array size.

692

693 Across trials within a problem, squirrel monkeys either did not improve their performance (with 694 the touchscreen, Experiment 1) or improved their performance modestly (with the 3D objects, 695 Experiment 2 , from $\sim 55 \%$ correct on $\mathrm{T} 2$ to $\sim 61 \%$ correct on $\mathrm{T} 5)$. These results may indicate that 696 squirrel monkeys need more than five trials' worth of experience to reliably locate the reward for any given stimulus array. (Harlow (1949), for example, gave monkeys between 6 and 50 trials for each problem; Darby and Riopelle (1955) gave macaques 6 trials per problem; and Kangas and Bergman (2014) gave squirrel monkeys 200 trials. However, it should be noted that in those paradigms, unlike the ones presented here, the stimuli changed position between trials.) Squirrel monkeys' relatively low problem-level competence in our paradigm would, in turn, make it difficult for them to learn the task-level contingencies.

The presentation medium (2D touchscreen or 3D object) had no main effect on either the monkeys' success on the task or their tendency to repeat a selection. Thus, we found no evidence 
706

707

708

709

710

711

712

713

714

715

716

717

718

719

720

721

722

723

724

725

726

727

728

to suggest that either task was better suited to the abilities of this population, despite the fact that presentation medium did interact with other variables (information type and source).

Squirrel monkeys exhibited a strong tendency to repeat their own selections, following both rewarded and unrewarded information trials. This resulted in unrewarded information trials having success rates below chance in the individual conditions. This tendency to repeat one's own previous selections has been documented by other researchers; notably, macaque monkeys, in an individual learning condition, frequently repeated their own selections, regardless of whether they had been rewarded for that selection or not (Monfardini et al., 2012).

However, even given this tendency, squirrel monkeys repeated their own selection more when that selection had resulted in a win than a lose trial. This result indicates some sensitivity to feedback, in that monkeys were more likely to repeat something that had resulted in either a reward or the appearance of secondary reinforcers (auditory click and sunburst image). A similar differential sensitivity to reward (versus nonreward) feedback has been reported for Japanese macaques (Itoh, Izumi, \& Kojima, 2001) as well as baboons and pigeons (Cook \& Fagot, 2009).

The effect of information source on repetition differed between the two presentation mediums. In the touchscreen task (Experiment 1), the tendency to repeat the selection made in the information trial was not different between the individual and social conditions. Monkeys' repeating behaviour did show an interaction that was near the alpha criterion threshold $(p=0.056)$ between information type and social demonstration type (human or puppet). This trend was in the direction of greater sensitivity to the win/lose distinction for the human's demonstration, 
729

730

731

732

733

734

735

736

737

738

739

740

741

742

743

744

745

746

747

748

749

750

751

compared with that of the puppet. This made it logical to use a human demonstrator only in

Experiment 2. While the value of puppet or otherwise artificial demonstrators in other contexts is clear (Krupenye et al., 2016; Truskanov \& Lotem, 2017; Wood, Kendal, \& Flynn, 2013), it was not beneficial for the monkeys' performance in this case.

In contrast, in the 3D object task (Experiment 2), there was a main effect of information source: monkeys tended to repeat their own selections more than those of the social demonstrator. In this Experiment, there was also a main effect of information type on the tendency to repeat, but no interaction between source and information type. This result indicates a sensitivity to source, and to whether a selection was rewarded, but no differential sensitivity to reward information based on the source.

Across both tasks (see combined analyses) the monkeys' tendency to repeat selections was greater in the individual condition than the social condition. In contrast, when children were given a similar task on a touchscreen with information acquired from either a social demonstration or individual exploration, they were largely unaffected by the information source (Atkinson et al., 2019). This is likely attributable to the fact that the children apparently understood the predictive value of the information trial (in both conditions), as evidenced by their higher success rates ( $\sim 73 \%$, c.f. $\sim 55 \%$ for the monkeys). Children's performance appeared to reflect the task-specific relevance of win and lose information trials, whereas (as previously noted) the monkeys' performance presumably reflects pre-existing biases in their behaviour (e.g. stimulus enhancement effects in the social condition, and possibly simple behavioural inertia in the individual condition). 
753 Because the squirrel monkeys did not reach high proficiency levels in our tasks, the results of

754 these experiments cannot be directly compared to results from human children, who

755 demonstrated high levels of task success (Atkinson et al., 2019). Such a comparison would have

756 the goal of illuminating the effects of information source across populations, with a view to

757 better understanding the uniqueness of human culture. Drawing comparisons with human

758 performance will require further study with NHP populations that learn to perform the task

759 proficiently. In our ongoing research, we have also tested capuchin monkeys with the

760 touchscreen task, with several individuals so far achieving above-criterion performance (Kean et

761 al., 2018).

762

763 What our results can reveal, however, is the biases that squirrel monkeys may have when approaching a task. The tendency of squirrel monkeys to repeat selections was not a trained response, and was similar for both mediums of presentation. We speculate that such biases in nonhuman behaviour could, in certain studies, generate patterns of behaviour that would be consistent with certain interpretations of social learning and/or cultural transmission (i.e. apparent "copying" of others' behaviour), in spite of the fact that this could be the outcome of mechanisms very different from those driving human social learning and cultural transmission. For example, following observation of an experienced individual, a previously naïve animal may perform the same foraging behaviour. This might occur in the absence of any understanding of either the goal of the demonstrator or even the effect of the behaviour (i.e. the connection with food), although the individual's behaviour would also be consistent with these higher-order cognitive explanations. 
776 In the current studies, our research designs have allowed us to directly compare responses to

777

778

779

780

781

782

783

784

785

786

787

788

789

790

791

792

793

794

795

796

797

social demonstrations with those following individual exploration, under equivalent conditions.

Furthermore, we were able to evaluate the extent to which the subjects were influenced by the outcome of the response observed or experienced. These comparisons illuminate the fact that the monkeys' slight bias to repeat the behaviour of others was both (a) less strong, if anything, than their tendency to repeat their own behaviour, and (b) not motivated by a recognition of the connection between the repeated response and its outcome (since this also occurred-even if to a slightly more limited extent—following unrewarded information trials). Despite our limited success in training the squirrel monkeys on this task, we believe that it is important for researchers interested in animal social learning to pursue designs such as ours, which allow direct comparisons between responses to information acquired from social demonstrations and (equivalent) information acquired from personal experience. Such research may shed valuable light on the mechanisms underlying effects of social influence.

\section{Acknowledgments}

We thank the Living Links to Human Evolution Research Centre staff at RZSS Edinburgh Zoo for their training and support throughout this study, and RZSS Edinburgh Zoo for facilitating this research. We are also grateful to Lindsay Young for her assistance with experimental materials for Experiment 2. 
798

799

800

801

802

803

804

805

806

807

808

809

810

811

812

813

814

815

816

817

818

819

820

\section{References}

Anderson, J. R., Kuroshima, H., \& Fujita, K. (2017). Observational learning in capuchin monkeys: a video deficit effect. The Quarterly Journal of Experimental Psychology, 70(7), 1254-1262. https://doi.org/10.1080/17470218.2016.1178312

Atkinson, M., Thompson, B., Renner, E., Mackintosh, G., Xie, D., Su, Y., \& Caldwell, C. A. (2019). Robust source-independent biases in children's use of socially and individually acquired information. [Abstract PS-2.27] Interdisciplinary Advances in Statistical Learning Conference, San Sebastián, Spain.

Barr, D. J., Levy, R., Scheepers, C., \& Tily, H. J. (2013). Random effects structure for confirmatory hypothesis testing: Keep it maximal. Journal of Memory and Language, 68, 255-278. doi:10.1016/j.jml.2012.11.001

Barr, R., \& Hayne, H. (1999). Developmental changes in imitation from television during infancy. Child Development, 70(5), 1067-1081. https://doi.org/10.1111/1467-8624.00079

Bates, D., Mächler, M., Bolker, B. M., \& Walker, S. C. (2015). Fitting linear mixed-effects models using lme4. Journal of Statistical Software, 67(1), 1-48. https://doi.org/10.18637/jss.v067.i01

Berman, P. W. (1971). Stimulus novelty as a variable in children's win-stay, lose-shift discrimination learning set. Child Development, 42, 1591-1595.

Berman, P. W., Rane, N. G., \& Bahow, E. (1970). Age changes in children's learning set with win-stay, lose-shift problems. Developmental Psychology, 2(2), 233-239.

Cook, R., \& Fagot, J. (2009). First trial rewards promote 1-trial learning and prolonged memory in pigeon and baboon. Proceedings of the National Academy of Sciences, 106(23), 9530- 
9533. https://doi.org/10.1073/pnas.0903378106

822 Csibra, G., \& Gergely, G. (2011). Natural pedagogy as evolutionary adaptation. Philosophical

823 Transactions of the Royal Society B: Biological Sciences, 366, 1149-1157.

824 https://doi.org/10.1098/rstb.2010.0319

825 Darby, C. L., \& Riopelle, A. J. (1955). Differential problem sequences and the formation of 826 learning sets. The Journal of Psychology, 39, 105-108.

827 Dean, L. G., Kendal, R. L., Schapiro, S. J., Thierry, B., \& Laland, K. N. (2012). Identification of

828 the social and cognitive processes underlying human cumulative culture. Science, 335, $1114-1118$.

830 Egelkamp, C. L., \& Ross, S. R. (2019). A review of zoo-based cognitive research using 831 touchscreen interfaces. Zoo Biology, 38, 220-235. https://doi.org/10.1002/zoo.21458

832 Fox, J. (2002). Fitting linear models. In An R and S-Plus Companion to Applied Regression (pp. 833 119-153). Thousand Oaks, CA: Sage Publications.

834 Guidelines for the treatment of animals in behavioural research and teaching. (2015). Animal Behaviour, 99, I-IX. https://doi.org/10.1016/S0003-3472(14)00451-5

836 Harlow, H. F. (1949). The formation of learning sets. Psychological Review, 56(1), 51-65. 837 https://doi.org/10.1037/h0062474

838 Horner, V., \& Whiten, A. (2005). Causal knowledge and imitation/emulation switching in 839 chimpanzees (Pan troglodytes) and children (Homo sapiens). Animal Cognition, 8(3), 164840 181. https://doi.org/10.1007/s10071-004-0239-6

841 Itoh, K., Izumi, A., \& Kojima, S. (2001). Object discrimination learning in aged Japanese 842 843 monkeys. Behavioral Neuroscience, 115(2), 259-270. https://doi.org/10.1037/07357044.115.2.259 
844 Kangas, B. D., \& Bergman, J. (2012). A novel touch-sensitive apparatus for behavioral studies in

845 unrestrained squirrel monkeys. Journal of Neuroscience Methods, 209(2), 331-336.

$846 \quad$ https://doi.org/10.1016/j.jneumeth.2012.06.028

847 Kangas, B. D., \& Bergman, J. (2014). Repeated acquisition and discrimination reversal in the 848 squirrel monkey (Saimiri sciureus). Animal Cognition, 17(2), 221-228.

$849 \quad$ https://doi.org/10.1007/s10071-013-0654-7

850 Kean, D., Renner, E., Atkinson, M., \& Caldwell, C. (2018). Capuchin monkeys can learn and

851 generalise a win-stay, lose-shift strategy under social information and individual exploration

852 conditions. [Abstract] Cultural Evolution Conference 2018 Abstracts: 36.

853 Krupenye, C., Kano, F., Hirata, S., Call, J., \& Tomasello, M. (2016). Great apes anticipate that

854 other individuals will act according to false beliefs. Science, 354(6308), 110-114.

855 Lenth, R., Singmann, H., Love, J., Buerkner, P., \& Herve, M. (2019). emmeans: Estimated

856 Marginal Means, aka Least-Squares means. Available at https://cran.r-

857 project.org/web/packages/emmeans/index.html

858 Levinson, B., \& Reese, H. W. (1967). Patterns of discrimination learning set in preschool

859 children, fifth-graders, college freshmen, and the aged. Monographs of the Society for

$860 \quad$ Research in Child Development, 32(7), 1-92.

861 Lyons, D. E., Young, A. G., \& Keil, F. C. (2007). The hidden structure of overimitation.

862 Proceedings of the National Academy of Sciences, 104(50), 19751-19756.

863 https://doi.org/10.1073/pnas.0704452104

864

MacDonald, C., \& Whiten, A. (2011). The “Living Links to Human Evolution” Research Centre in Edinburgh Zoo: A new endeavour in collaboration. International Zoo Yearbook, 45(1), 7-17. https://doi.org/10.1111/j.1748-1090.2010.00120.x 
867 Monfardini, E., Gaveau, V., Boussaoud, D., Hadj-Bouziane, F., \& Meunier, M. (2012). Social

868 learning as a way to overcome choice-induced preferences? Insights from humans and

869 rhesus macaques. Frontiers in Neuroscience, 6, 127.

870 https://doi.org/10.3389/fnins.2012.00127

871 Monfardini, E., Hadj-Bouziane, F., \& Meunier, M. (2014). Model-observer similarity, error

872 modeling and social learning in rhesus macaques. PLoS ONE, 9(2), e89825.

873 https://doi.org/10.1371/journal.pone.0089825

Peirce, J., Gray, J. R., Simpson, S., MacAskill, M., Höchenberger, R., Sogo, H., ... Lindeløv, J.

875 K. (2019). PsychoPy2: Experiments in behavior made easy. Behavior Research Methods, 51, 195-203. https://doi.org/10.3758/s13428-018-01193-y

Pryor, K. (2002). Don't shoot the dog! The new art of teaching and training (Revised Ed). Dorking, Surrey, UK: Ringpress Books Ltd.

R Core Team. (2017). R: A language and environment for statistical computing. Vienna, Austria: R Foundation for Statistical Computing. Retrieved from https://www.r-project.org/

Renner, E. (2015). Social and Asocial Learning in Humans and Orangutans. D. Phil. Thesis, The George Washington University.

Schad, D. J., Hohenstein, S., Vasishth, S., \& Kliegl, R. (2018). How to capitalize on a priori contrasts in linear (mixed) models: A tutorial. Eprint ArXiv:1807.10451. Available at https://arxiv.org/abs/1807.10451 (accessed 15 November 2018)

Templeton, J. J. (1998). Learning from others' mistakes: a paradox revisited. Animal Behaviour, 887 55(1), 79-85. https://doi.org/10.1006/anbe.1997.0587

Tennie, C., Call, J., \& Tomasello, M. (2009). Ratcheting up the ratchet: on the evolution of 889 cumulative culture. Philosophical Transactions of the Royal Society B: Biological Sciences, 
891 The British Psychological Society. (2012). Guidelines for Psychologists Working with Animals.

892 Leicester. Available at https://www.bps.org.uk/news-and-policy/bps-guidelines-

893 psychologists-working-animals (accessed 14 June 2019)

894 Tomasello, M., Kruger, A. C., \& Ratner, H. H. (1993). Cultural learning. Behavioral and Brain

$895 \quad$ Sciences, 16(03), 495-552. https://doi.org/10.1017/S0140525X00031496

896 Truskanov, N., \& Lotem, A. (2017). Trial-and-error copying of demonstrated actions reveals

897 how fledglings learn to "imitate" their mothers. Proceedings of the Royal Society B:

898 Biological Sciences, 284, 20162744. https://doi.org/10.1098/rspb.2016.2744

899 Waitt, C., \& Buchanan-Smith, H. M. (2006). Perceptual considerations in the use of colored

900 photographic and video stimuli to study nonhuman primate behavior. American Journal of

901 Primatology, 68, 1054-1067. https://doi.org/10.1002/ajp

902 Whiten, A., Allan, G., Devlin, S., Kseib, N., Raw, N., \& McGuigan, N. (2016). Social learning in 903 the real-world: "Over-imitation" occurs in both children and adults unaware of participation 904 in an experiment and independently of social interaction. PLoS ONE, 11(7), 1-14.

905 https://doi.org/10.1371/journal.pone.0159920

906 Wood, L. A., Kendal, R. L., \& Flynn, E. G. (2013). Copy me or copy you? The effect of prior 907 experience on social learning. Cognition, 127(2), 203-213.

908 https://doi.org/10.1016/j.cognition.2013.01.002

909 
910 Figure Legends

911

912 Figure 1. Touchscreen example problem and apparatus setup. (A) A sample two-stimulus (Stage

913 A) problem. (B) Appearance of the screen (example) after the rewarded stimulus was selected,

914 with the reward cue (sunburst) appearing at the location of the rewarded stimulus. (C) A squirrel

915 monkey interacting with the touchscreen task.

916

917 Figure 2. The squirrel monkey puppet used as a demonstrator in Experiment 1.

918

919 Figure 3. The effects of source and information type on whether (in T2) squirrel monkeys

920 repeated the selection from the information trial. Results are from the touchscreen task of

921 Experiment 1. Each point represents the aggregate performance of an individual monkey in the

922 given condition, and the size of each point indicates the number of trials aggregated within that

923 mean. Boxes and whiskers indicate medians and interquartile ranges of performance aggregated

924 by monkey.

925

926 Figure 4. Success across experimental trials in the touchscreen task of Experiment 1. Plotted are

927 the means of by-subject means for illustration purposes; statistical tests did not average subject

928 means. The error bars indicate $95 \%$ confidence intervals on the means of means. Statistical tests

929 revealed a non-significant trend towards improvement across trials.

930

931 Figure 5. Probability of repeating in T2 the selection made on the information trial, according to

932 information type, for Stage A and Stage B, for the monkey who passed criterion (Amarilla). 
933 Amarilla was in the individual condition. Expected rates of repetition according to chance are

934 indicated by the horizontal lines (separately for each of the stages).

935

936 Figure 6. Two of the cups and felt sleeves in front of the occluder used in Experiment 2.

937

938 Figure 7. The effects of source and information type on whether (in T2) squirrel monkeys

939 repeated the selection from the information trial. Results are from the 3D object task of

940 Experiment 2. Each point represents the aggregate performance of an individual monkey in the

941 given condition, and the size of each point indicates the number of trials aggregated within that

942 mean. Boxes and whiskers indicate medians and interquartile ranges of performance aggregated

943 by monkey.

944

945 Figure 8. Success across experimental trials in the 3D object task of Experiment 2. Plotted are

946 the means of by-subject means for illustration purposes; statistical tests did not average subject

947 means. The error bars indicate $95 \%$ confidence intervals on the means of means. Statistical tests

948 revealed a significant effect of trial number.

949

950 Figure 9. The effects of presentation medium, source, and information type on whether squirrel

951 monkeys repeated the selection from the information trial on T2. Each point represents the

952 aggregate performance of an individual monkey, and the size of each point indicates the number

953 of trials aggregated within that mean. Boxes and whiskers indicate medians and interquartile

954 ranges of performance aggregated by monkey. 
Table $\mathbf{1}$ (on next page)

Participant characteristics 
1 Table 1. Participant characteristics

\begin{tabular}{|c|c|c|c|c|c|c|c|c|}
\hline Name & Sex & Group & $\begin{array}{l}\text { Age } \\
\text { (as of } \\
\text { Feb. } \\
2016 \text { ) }\end{array}$ & Condition & $\begin{array}{l}\text { Participation } \\
\text { in Expt } 1\end{array}$ & $\begin{array}{l}\text { No. } \\
\text { sessions, } \\
\text { Expt } 1\end{array}$ & $\begin{array}{l}\text { Participation } \\
\text { in Expt } 2\end{array}$ & $\begin{array}{l}\text { No. } \\
\text { sessions, } \\
\text { Expt } 2\end{array}$ \\
\hline Amarilla & $\mathrm{F}$ & East & 4 & Individual & $\begin{array}{l}\text { Training, } \\
\text { testing }\end{array}$ & $\begin{array}{l}\text { A: } 44 \\
B: 19\end{array}$ & $\begin{array}{l}\text { Testing } \\
\text { (insufficient } \\
\text { sessions) }\end{array}$ & 9 \\
\hline Boa & $\mathrm{M}$ & East & 10 & $\mathrm{~N} / \mathrm{A}$ & Training & 0 & $\mathrm{~N} / \mathrm{A}$ & 0 \\
\hline Cali & $\mathrm{F}$ & East & 10 & Social & $\begin{array}{l}\text { Training, } \\
\text { testing }\end{array}$ & 42 & Testing & 20 \\
\hline Ciara & $\mathrm{F}$ & East & 4 & Social & $\begin{array}{l}\text { Training, } \\
\text { testing }\end{array}$ & 55 & Testing & 19 \\
\hline Dora & $F$ & East & 5 & Individual & $\begin{array}{l}\text { Training, } \\
\text { testing }\end{array}$ & 69 & Testing & 20 \\
\hline Elie & $\mathrm{F}$ & East & 10 & Individual & Training & 0 & Testing & 15 \\
\hline Flora & $\mathrm{F}$ & East & 7 & Individual & $\begin{array}{l}\text { Training, } \\
\text { testing }\end{array}$ & 34 & $\begin{array}{l}\text { Testing } \\
\text { (insufficient } \\
\text { sessions) }\end{array}$ & 3 \\
\hline Gabriela & $\mathrm{F}$ & East & 4 & Social & $\begin{array}{l}\text { Training, } \\
\text { testing }\end{array}$ & 61 & Testing & 20 \\
\hline Gisele & $\mathrm{F}$ & West & 3 & Social & $\begin{array}{l}\text { Training, } \\
\text { testing }\end{array}$ & 27 & $\begin{array}{l}\text { Testing } \\
\text { (insufficient } \\
\text { sessions) }\end{array}$ & 0 \\
\hline Jasmin & $\mathrm{F}$ & West & 13 & Individual & $\begin{array}{l}\text { Training, } \\
\text { testing }\end{array}$ & 14 & Testing & 14 \\
\hline Lexi & $\mathrm{F}$ & East & 5 & Social & Training & 0 & $\begin{array}{l}\text { Testing } \\
\text { (insufficient } \\
\text { sessions) }\end{array}$ & 4 \\
\hline Loki & $\mathrm{F}$ & West & 1 & Social & $\mathrm{N} / \mathrm{A}$ & 0 & Testing & 15 \\
\hline Maya & $\mathrm{F}$ & East & 12 & $\mathrm{~N} / \mathrm{A}$ & Training & 0 & $\mathrm{~N} / \mathrm{A}$ & 0 \\
\hline Orla & $\mathrm{F}$ & West & 4 & Individual & $\begin{array}{l}\text { Training, } \\
\text { testing }\end{array}$ & 52 & $\begin{array}{l}\text { Testing } \\
\text { (insufficient } \\
\text { sessions) }\end{array}$ & 0 \\
\hline Pelusa & $\mathrm{F}$ & East & 4 & Individual & $\begin{array}{l}\text { Training, } \\
\text { testing }\end{array}$ & 60 & Testing & 20 \\
\hline Roca & $\mathrm{F}$ & East & 13 & Individual & Training & 0 & $\begin{array}{l}\text { Testing } \\
\text { (insufficient } \\
\text { sessions) }\end{array}$ & 9 \\
\hline Sancha & $F$ & West & 6 & Social & $\begin{array}{l}\text { Training, } \\
\text { testing }\end{array}$ & 67 & Testing & 20 \\
\hline Sipi & $\mathrm{F}$ & East & 6 & Social & Training & 0 & $\begin{array}{l}\text { Testing } \\
\text { (insufficient } \\
\text { sessions) }\end{array}$ & 3 \\
\hline Tatu & $\mathrm{F}$ & East & 14 & Social & Training & 0 & Testing & 1 \\
\hline
\end{tabular}




\begin{tabular}{l|l|l|l|l|l|r|l|c}
\hline & & & & & & & $\begin{array}{l}\text { (insufficient } \\
\text { sessions) }\end{array}$ & \\
\hline Toomi & F & West & 9 & Individual & $\begin{array}{l}\text { Training, } \\
\text { testing }\end{array}$ & $64 \begin{array}{l}\text { Testing } \\
\text { (side bias) }\end{array}$ & 12 \\
\hline Valencia & F & East & 4 & Social & $\begin{array}{l}\text { Training, } \\
\text { testing }\end{array}$ & 59 & Testing & 16 \\
\hline Yendi & F & East & 7 & N/A & Training & 0 & N/A & 0 \\
\hline
\end{tabular}

2 Expt, Experiment; F, female; M, male. For Experiment 1, completion of at least one T2 was

3 required for the data from a session to be used. For Experiment 2, completion of at least one full

4 problem (T1-T5) was required for the data from a session to be used. For Experiment 2, the data

5 from monkeys who completed fewer than 10 sessions, indicated by "(insufficient sessions)",

6 were not included in the analysis. 
Table 2 (on next page)

Performance summary (all trials, un-averaged) for Experiments 1 and 2 
1 Table 2. Performance summary (all trials, un-averaged) for Experiments 1 and 2

\begin{tabular}{|l|l|l|}
\hline Study and Condition & T2 success after win trials (\%) & T2 success after lose trials (\%) \\
\hline Tablet (Stage A) & & \\
\hline Social: human & $70^{*}$ & 50 \\
\hline Social: puppet & $62^{*}$ & 47 \\
\hline Individual & $70^{*}$ & $40 \dagger$ \\
\hline Overall & $68^{*}$ & $44 \dagger$ \\
\hline 3D & & \\
\hline Social: human & 54 & 55 \\
\hline Individual & $79^{*}$ & $31 \dagger$ \\
\hline Overall & $64^{*}$ & 46 \\
\hline
\end{tabular}

$2 *$, performance significantly above chance; $\uparrow$, performance significantly below chance. Statistical 3 tests were two-tailed binomial tests against 50\% (chance) performance.

4

5 


\section{Table 3 (on next page)}

Model results summary for Experiments 1 and 2

Only models that assessed performance as measured by T2 success in Stage A are included in this Table. GLMMs included random effects not shown here as well as the fixed effects detailed in the Table; please see text for details. N.S., non-significant; ndf, not different from. 
1 Table 3. Model results summary for Experiments 1 and 2

\begin{tabular}{|c|c|c|c|}
\hline Analysis & Experiment & $\begin{array}{l}\text { Variable(s) } \\
\text { included in the } \\
\text { model }\end{array}$ & $\begin{array}{l}p \text { value and direction } \\
\text { of effect }\end{array}$ \\
\hline \multirow{2}{*}{$\begin{array}{l}\text { Effect of session } \\
\text { number }\end{array}$} & Experiment 1 (tablet) & Session number & N.S., $p=0.47$ \\
\hline & Experiment 2 (3D) & Session number & N.S., $p=0.12$ \\
\hline \multirow{2}{*}{$\begin{array}{l}\text { Effect of trial } \\
\text { number }\end{array}$} & Experiment 1 (tablet) & Trial number & N.S., $p=0.064$ \\
\hline & Experiment 2 (3D) & Trial number & $\begin{array}{l}p=0.020 \\
\text { (later }>\text { earlier) }\end{array}$ \\
\hline \multirow{6}{*}{$\begin{array}{l}\text { Effect of source } \\
\text { and information } \\
\text { type }\end{array}$} & \multirow{3}{*}{ Experiment 1 (tablet) } & Source & $\begin{array}{l}p=0.043 \\
\text { (human }>\text { puppet) } \\
\text { N.S., } p=0.61 \\
\text { (social ndf individual) }\end{array}$ \\
\hline & & Information type & $p<0.001$ (win $>$ lose $)$ \\
\hline & & $\begin{array}{l}\text { Source * information } \\
\text { type }\end{array}$ & $\begin{array}{l}p=0.002 \\
\text { (see Supplemental } \\
\text { Table S2) }\end{array}$ \\
\hline & \multirow{3}{*}{ Experiment 2 (3D) } & Source & $\begin{array}{l}\text { N.S., } p=0.66 \\
\text { (social ndf individual) }\end{array}$ \\
\hline & & Information type & $p<0.001$ (win $>$ lose $)$ \\
\hline & & $\begin{array}{l}\text { Source * information } \\
\text { type }\end{array}$ & $\begin{array}{l}p<0.001 \\
\text { (see Supplemental } \\
\text { Table S3) }\end{array}$ \\
\hline
\end{tabular}

Only models that assessed performance as measured by T2 success in Stage A are included in

3 this Table. GLMMs included random effects not shown here as well as the fixed effects detailed

4 in the Table; please see text for details. N.S., non-significant; ndf, not different from. 


\section{Figure 1}

Touchscreen example problem and apparatus setup.

(A) A sample two-stimulus (Stage A) problem. (B) Appearance of the screen (example) after the rewarded stimulus was selected, with the reward cue (sunburst) appearing at the location of the rewarded stimulus. (C) A squirrel monkey interacting with the touchscreen task.

A

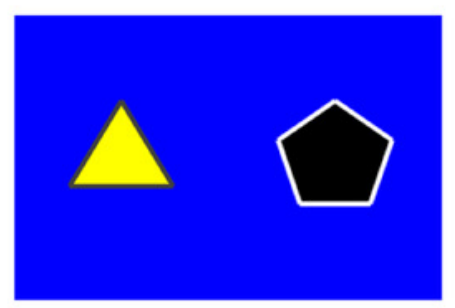

C

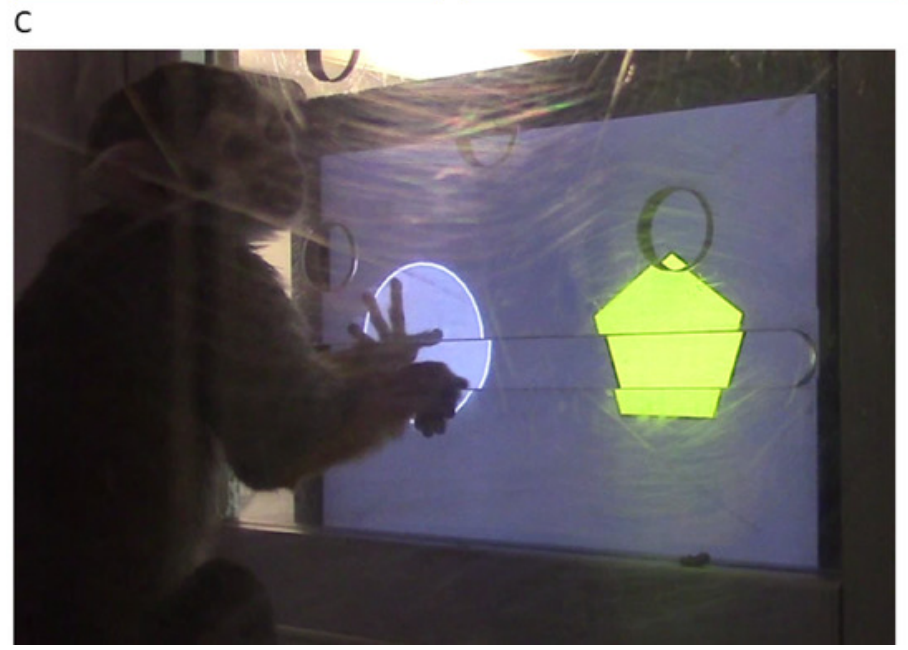


Figure 2

The squirrel monkey puppet used as a demonstrator in Experiment 1. 


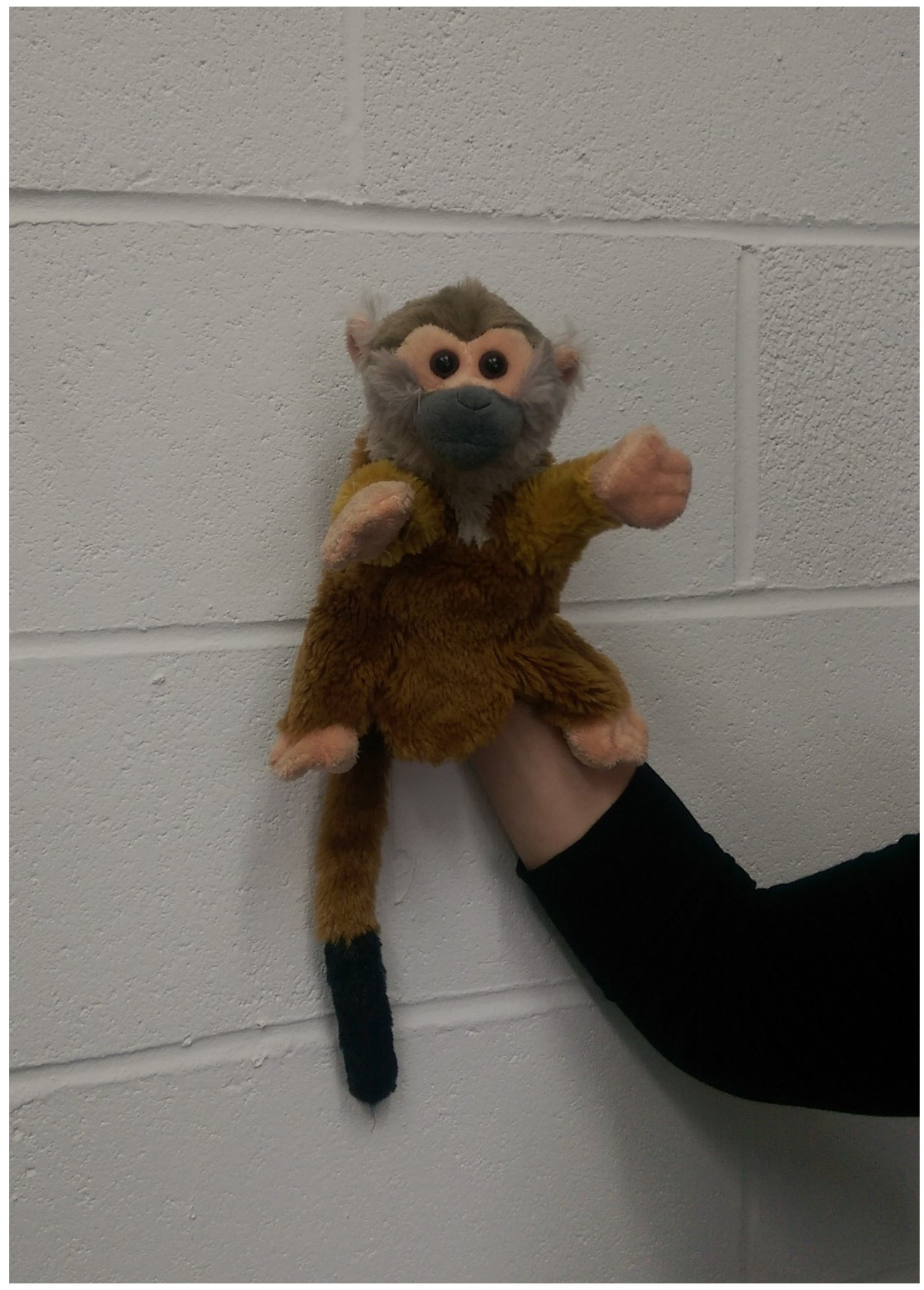




\section{Figure 3}

The effects of source and information type on whether (in T2) squirrel monkeys repeated the selection from the information trial.

Results are from the touchscreen task of Experiment 1. Each point represents the aggregate performance of an individual monkey in the given condition, and the size of each point indicates the number of trials aggregated within that mean. Boxes and whiskers indicate medians and interquartile ranges of performance aggregated by monkey.
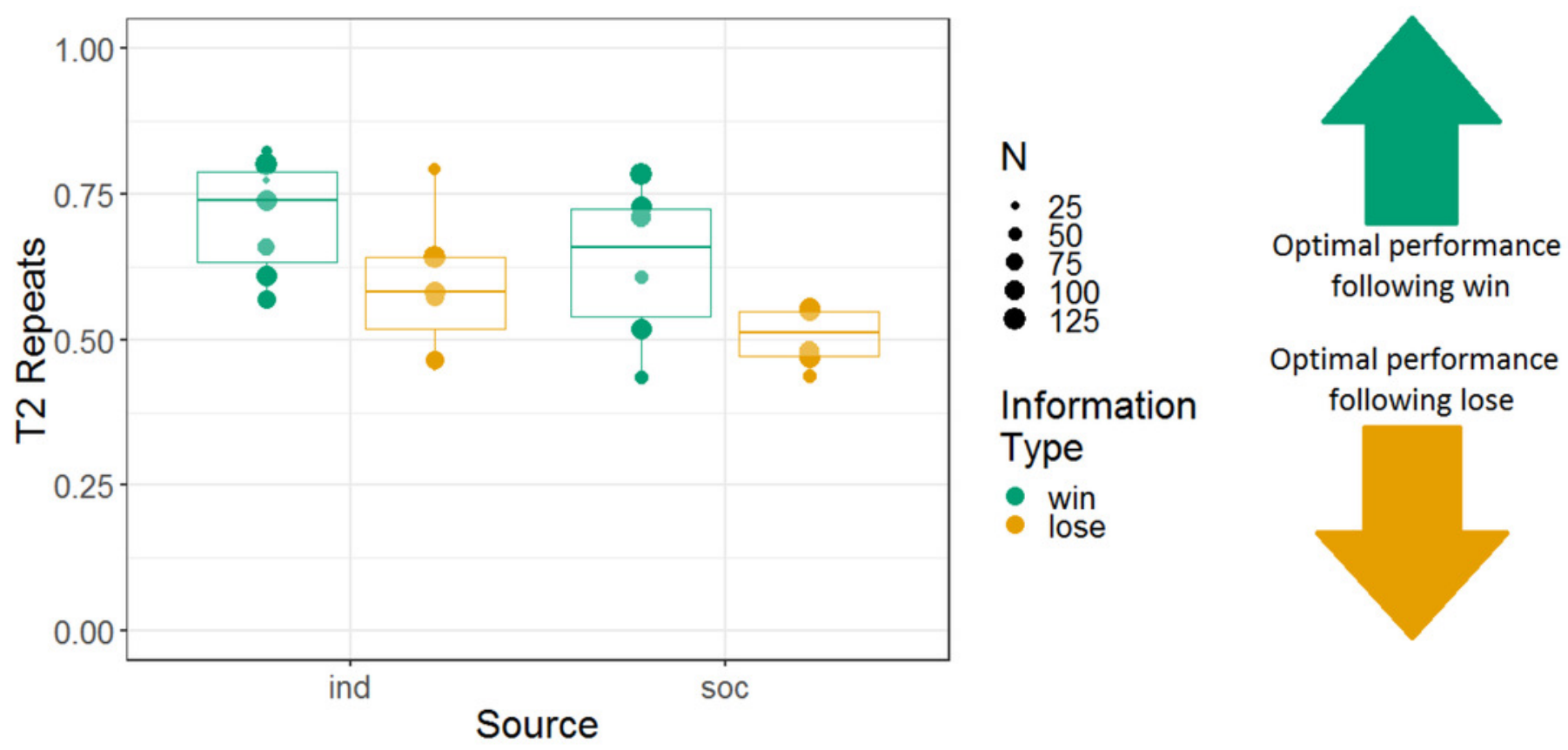


\section{Figure 4}

Success across experimental trials in the touchscreen task of Experiment 1.

Plotted are the means of by-subject means for illustration purposes; statistical tests did not average subject means. The error bars indicate $95 \%$ confidence intervals on the means of means. Statistical tests revealed a non-significant trend towards improvement across trials.

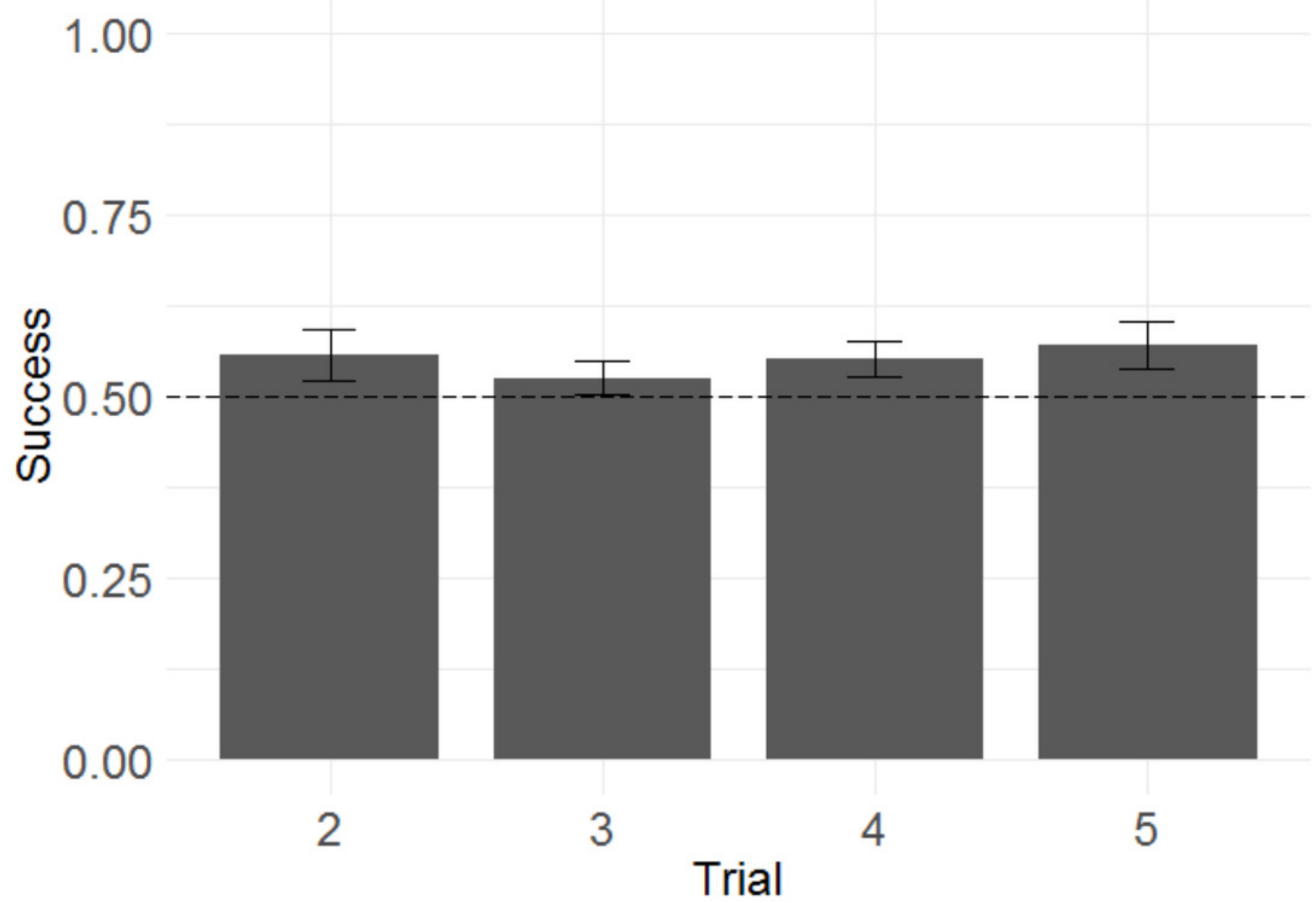




\section{Figure 5}

Probability of repeating in $\mathrm{T} 2$ the selection made on the information trial, according to information type, for Stage A and Stage B, for the monkey who passed criterion (Amarilla).

Amarilla was in the individual condition. Expected rates of repetition according to chance are indicated by the horizontal lines (separately for each of the stages).

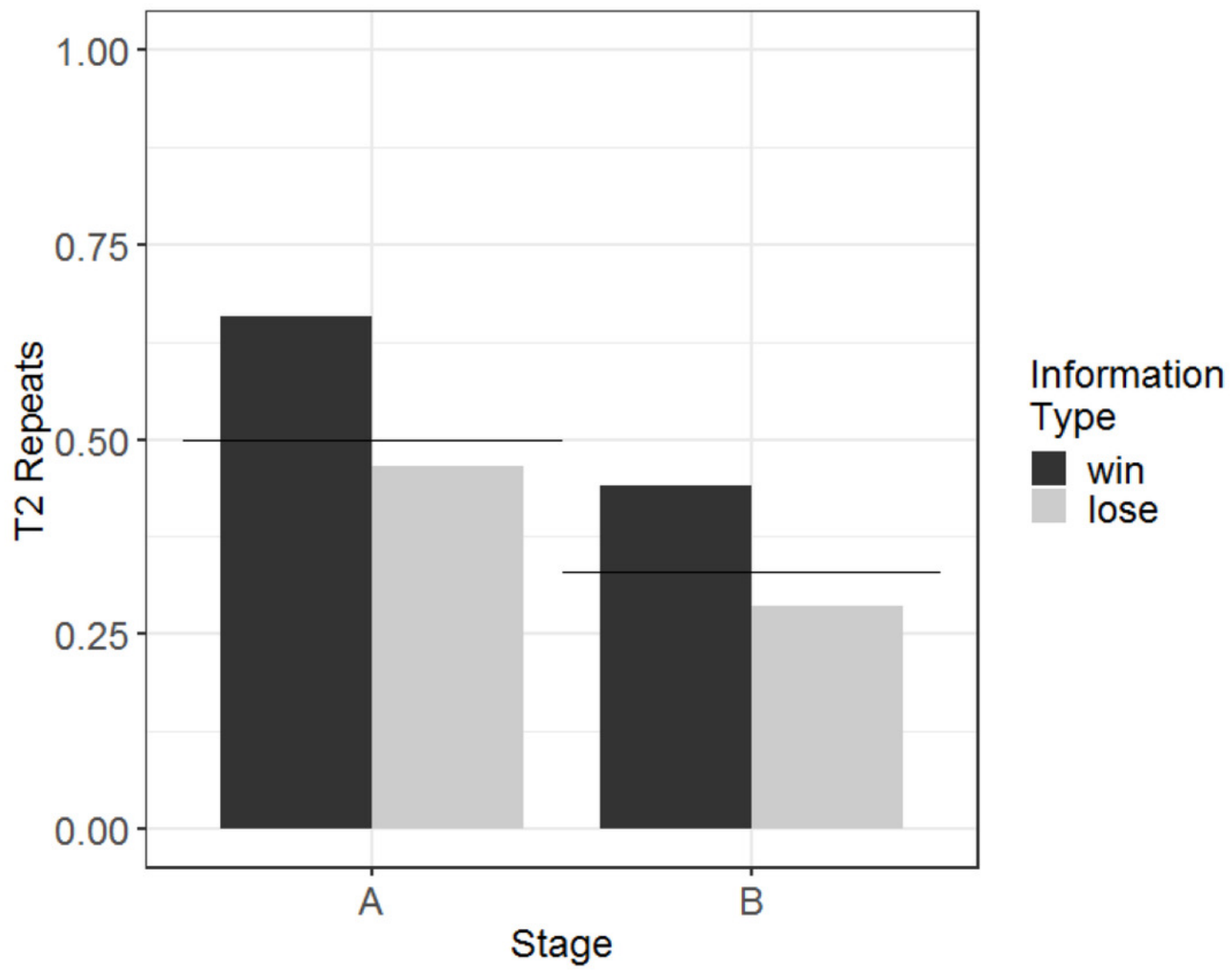




\section{Figure 6}

Two of the cups and felt sleeves in front of the occluder used in Experiment 2.

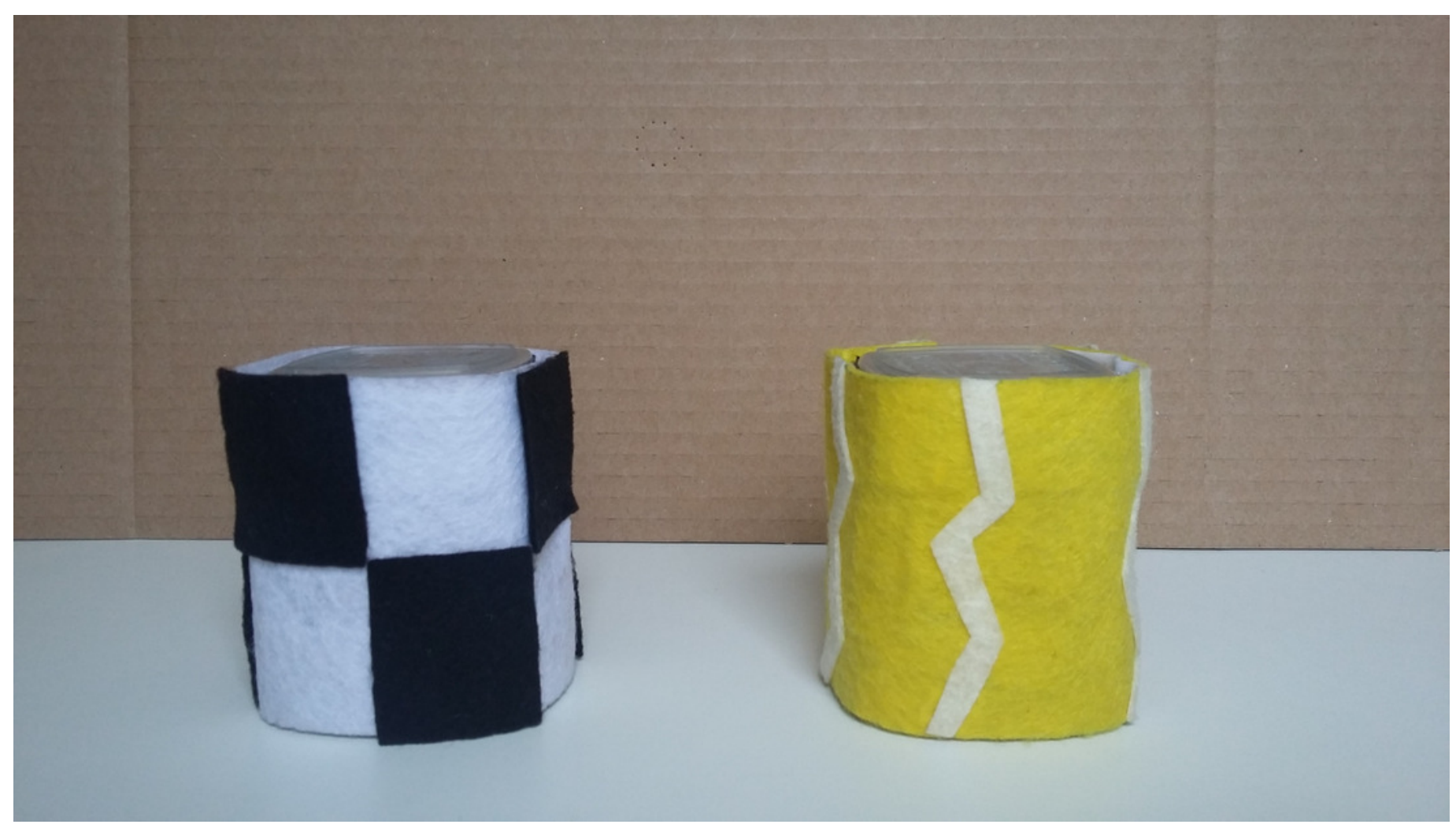




\section{Figure 7}

The effects of source and information type on whether (in T2) squirrel monkeys repeated the selection from the information trial.

Results are from the 3D object task of Experiment 2. Each point represents the aggregate performance of an individual monkey in the given condition, and the size of each point indicates the number of trials aggregated within that mean. Boxes and whiskers indicate medians and interquartile ranges of performance aggregated by monkey.

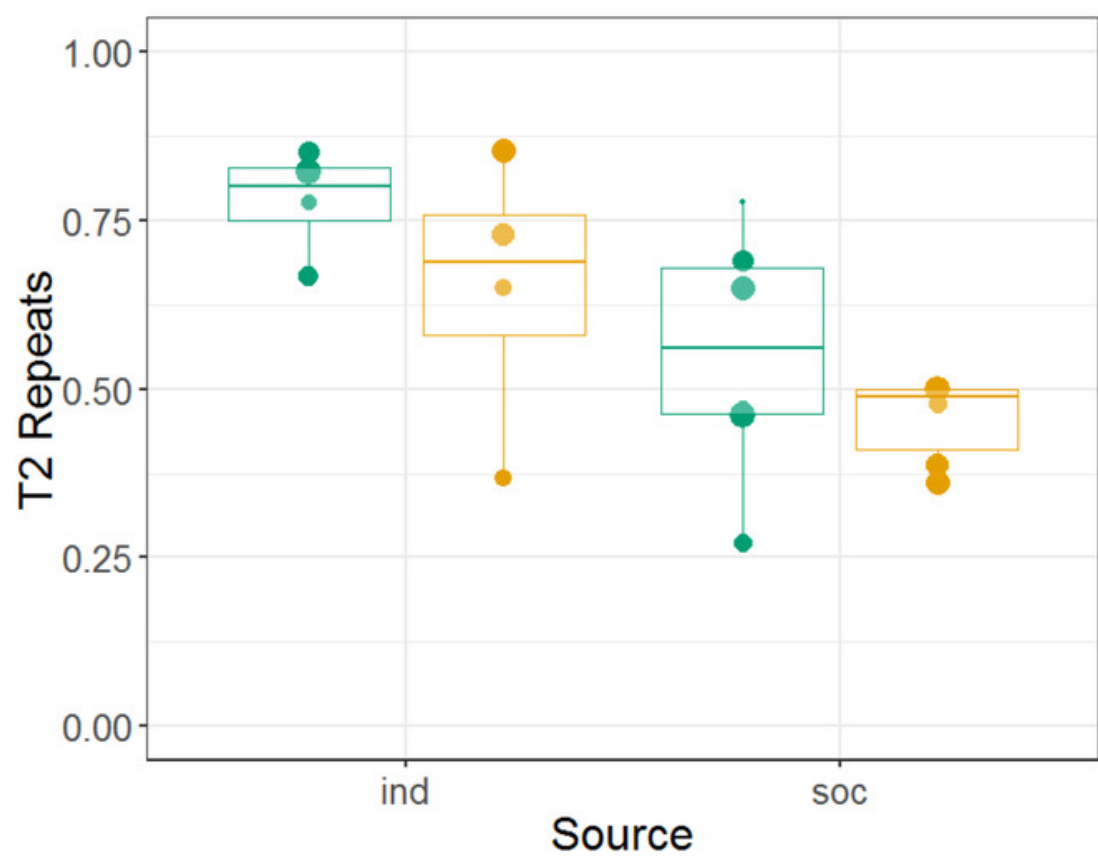

$\mathrm{N}$

- 10

- 20

- 30

Information

Type

win
lose

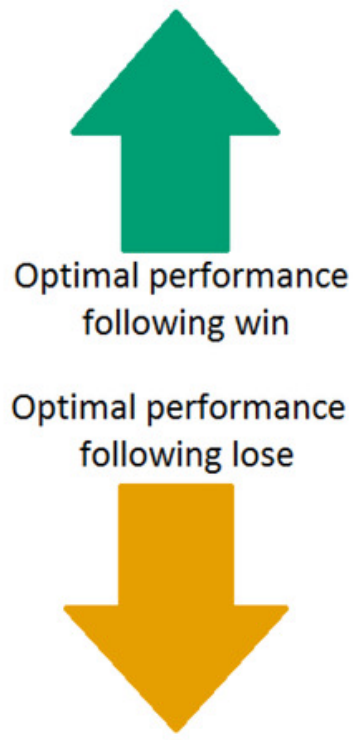




\section{Figure 8}

Success across experimental trials in the 3D object task of Experiment 2.

Plotted are the means of by-subject means for illustration purposes; statistical tests did not average subject means. The error bars indicate $95 \%$ confidence intervals on the means of means. Statistical tests revealed a significant effect of trial number.

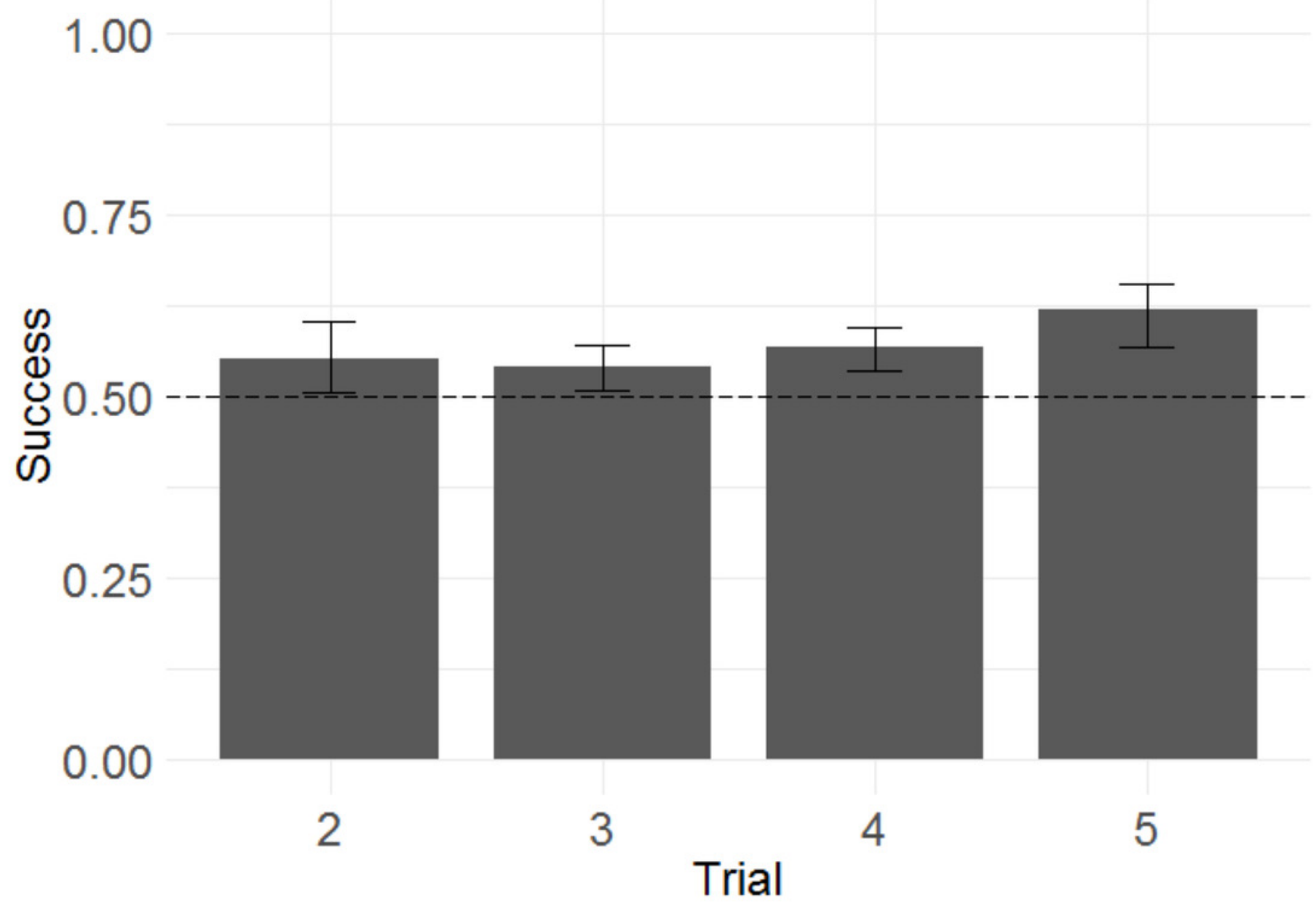




\section{Figure 9}

The effects of presentation medium, source, and information type on whether squirrel monkeys repeated the selection from the information trial on $\mathrm{T} 2$.

Each point represents the aggregate performance of an individual monkey, and the size of each point indicates the number of trials aggregated within that mean. Boxes and whiskers indicate medians and interquartile ranges of performance aggregated by monkey.
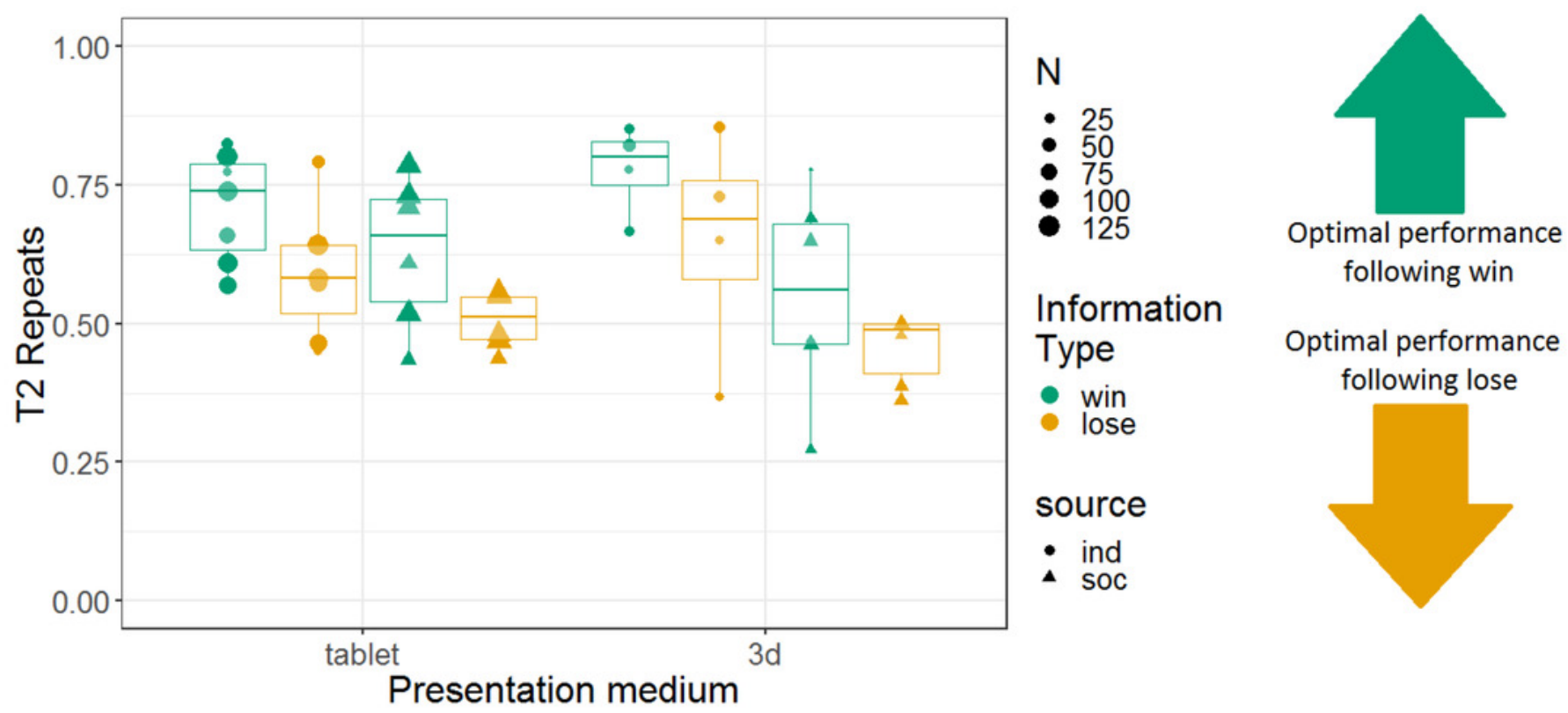

Optimal performance following lose

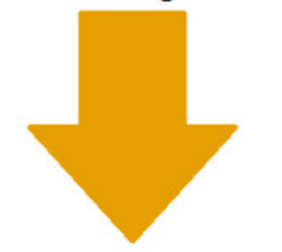

